\title{
First Quantum Corrections to the Classieal Partition Function for a Non-Relativistic Electrodynamical System
}

\author{
F. Ruiz Ruiz and R. F. Alvarez-Estrada*) \\ Departamento de Física Teórica, Facultad de Ciencias Físicas, Universidad Complutense, \\ 29040-Madrid, Spain
}

\begin{abstract}
The elassical partition function for a system in thermodynamical equilibrium formed by $N$ identical non-relativistic particles interacting through Coulomb potentials and with the dynamical electromagnetic field is studied. It is proved that the dynamical or transverse EM degrees of freedom decouple from the particle ones. It is also shown that this decoupling does to take place in the quantum mechanical partition function. The leading quantum corrections to the classical partition function are explicitly given. Such corrections are shown to be determined by instantaneous dipole-dipole coulombic interactions and by self-energy effects, and to receive no contribution from the interaction among different particles mediated by the dynamical EM field.
\end{abstract}

\section{Introduction}

The problem of the classical limit can be stated as that of proving that any quantum theory must tend to its classical version as the limit $h \rightarrow 0$ is taken. It has no simple solution, even in the case of a particle interacting with the dynamical EM field, as BIALYIICKI-BIRULA [1] has pointed out. To treat this case coherent states have been used [1]. More explicitly, it can be proved [2] that by means of a unitary transformation, related to coherency in some sense, the classical energy is the leading, $\hbar^{0}$-term, of the quantum energy. Among the various contributions to the classical limit problem in Quantum Electrodynamics (QED) we shall recall those of Dente [3], Stapp [4], Halpern and Siegel [5], and RohrLich [6] and references therein.

From the Statistical Mechanics viewpoint, the problem of the classical limit is still more interesting for two reasons. Firstly, because it is related to QED (or, even, to the more general problem of quantum field theories) at finite temperature $[7,8]$, so important is cosmological problems. And, secondly, because it corresponds to the much more physical situation of many charged particles.

The methods to be used in the study of Statistical Mechanics for QED consist in the evaluation of the partition function, namely, a trace. Each one of the matrix elements entering in the trace is evaluated by means of the path integral formalism. For an exposition of this formalism see FEYNMAN and HibBs [9, 10]. A more mathematical presentation is given by Srmon [11] and ThIRRING [12]. This paper is devoted to the analysis of the classical limit problem for many particle electrodynamical systems and essential differences are found between the classical and quantum statistics.

*) Address for the academic year 1985-86: High Energy Physics Group, Lawrence Berkeley Laboratory, Berkeley University, California, USA. 
It should be noticed that the Statistical Mechanics of and electrodynamical system is closely related to the physics of a plasma. Standard methods to study the latter, discussed by BaLescu [13] or IchImaru [14], could have been used. But for the study of the classical limit and the related problems treated in this paper the path integral formalism turns out to be more useful.

In section 2, we study a classical system in thermodynamical equilibrium formed by identical particles interacting with the dynamical EM field. It is proved that the transverse or dynamical degrees of freedom of the EM field decouple from the particle ones. The consequences of this fact on the mean values for the EM potential $\vec{A}$, the physical electric $\vec{E}$ and magnetic $\vec{B}$ fields and for the energy are derived. In section 3, the system of section 2 is studied quantum-mechanically by means of Feynman path integrals. As an aside (appendix $A$ ), we also find a bound for the quantum partition function for one particle interacting with the dynamical EM field. Section 4 is devoted to prove that when the limit is taken in the quantum partition function, the classical one is recovered. We also undertake the much more difficult task of evaluating the leading quantum corrections. Explicit expression for them are given, which constitute the main result of this work and, to our knowledge, are new. In section $\mathbf{5}$ another method of evaluating the classical limit is studied, namely, the one called quasiconstant field approximation.

\section{Classical Statistical Mechanies for Non-Relativistic Electrodynamies}

We consider $N$ classical (Cl) identical charged particles of charge $q$ and mass $m$ interacting with the classical EM field $\vec{A}_{\mathrm{Cl}}(\vec{x}, t)$. To avoid any stability problem we assume that there is a homogeneous background with charge of opposite sign to that of the particles and without any dynamical degrees of freedom, so that the system is electrically neutral. The Hamiltonian in the Coulomb gauge $\left(\vec{V} \vec{A}_{\mathrm{C} 1}(\vec{x}, t)=0\right)$ is given by

$$
\begin{aligned}
& H_{\mathrm{Cl}}=H_{p, \mathrm{Cl}}+H_{\mathrm{EM}, \mathrm{Cl}}+H_{c}, \quad H_{p, \mathrm{Cl}}=\frac{1}{2 m} \sum_{i=1}^{N}\left[\vec{p}_{i}-\frac{q}{c} \vec{A}_{\mathrm{Cl}}\left(\vec{x}_{i}, t\right)\right]^{2} \\
& H_{\mathrm{EM}, \mathrm{Cl}}=\frac{1}{2} \int d^{3} \vec{x}\left[\frac{1}{c^{2}}\left(\frac{\partial \vec{A}_{\mathrm{Cl}}}{\partial t}\right)^{2}+\left(\overrightarrow{\vec{V}} \wedge \vec{A}_{\mathrm{Cl}}\right)^{2}\right], \quad H_{c}=\sum_{i>j=1}^{N} \frac{q^{2}}{4 \pi\left|\vec{x}_{i}-\vec{x}_{j}\right|},
\end{aligned}
$$

where $\vec{x}_{i}$ and $\vec{p}_{i}$ are the position and momentum of the $i$ th particle and the integration over $\vec{x}$ is extended to the volume $V$ ocuppied by the system. Notice that the electrostatic (Coulomb) self-energy $U_{c}$ for each particle will be discarded both in the classical and quantum cases. Their inclusion would amount to an overall constant factor $\exp \left(-\beta N U_{c}\right)$ in the partition function. In a linear polarization basis and as $V \rightarrow \infty, \vec{A}_{\mathrm{Cl}}(\vec{x}, t)$ takes the form

$$
\vec{A}_{\mathrm{Cl}}(\vec{x}, t)=\sum_{\lambda=1,2} \int \frac{d^{3} \vec{k}}{(2 \pi)^{3 / 2}}\left[c(\vec{k}) \vec{\varepsilon}(\vec{k}, \lambda) a(\vec{k}, \lambda, t) e^{i \vec{k} \vec{x}}+\text { c.c. }\right],
$$

where we have introduced the classical complex amplitudes $a(\vec{k}, \lambda, t)(\lambda=1,2)$ and an ultraviolet cut-off factor $c(\vec{k}),\left(c(\vec{k})=c^{*}(-\vec{k})\right)$.

Using (complex) oscillator coordinates and momenta $q(\vec{k}, \lambda, t)$ and $\pi(k, \lambda, t)$ for each mode $(\vec{k}, \lambda)[15]$

$$
\begin{aligned}
& q(\vec{k}, \lambda, t)=\frac{i}{c}\left[a^{*}(-\vec{k}, \lambda, t)-a(\vec{k}, \lambda, t)\right], \\
& \pi(\vec{k}, \lambda, t)=-\frac{\omega}{c}\left[a^{*}(\vec{k}, \lambda, t)+a(-\vec{k}, \lambda, t)\right],
\end{aligned}
$$


with $q^{*}(\vec{k}, \lambda, t)=q(-\vec{k}, \lambda, t)$ and $\pi^{*}(\vec{k}, \lambda, t)=\pi(-\vec{k}, \lambda, t)$, the first Eq. (2.2) and Eq. (2.3) become

$$
\begin{aligned}
& H_{\mathrm{EM}, \mathrm{Cl}}=\frac{1}{2} \sum_{\lambda=1,2} \int \frac{d^{3} \vec{k}}{(2 \pi)^{3}}|c(\vec{k})|^{2}\left[|\pi(\vec{k}, \lambda, t)|^{2}+\omega^{2}|q(\vec{k}, \hat{\lambda}, t)|^{2}\right], \\
& \vec{A}_{\mathrm{C} 1}(\vec{x}, t)=i c \sum_{\lambda=1,2} \int \frac{d^{3} \vec{k}}{(2 \pi)^{3 / 2}} c(\vec{k}) \vec{\varepsilon}(\vec{k}, \lambda) q(\vec{k}, \lambda, t) e^{i \vec{k} \vec{x}} .
\end{aligned}
$$

The classical partition function in the canonical ensemble reads $([13,16])$

$$
Z_{N, \mathrm{Cl}}=\frac{1}{N !} \int \prod_{i=1}^{N} \frac{d^{3} \vec{x}_{i} d^{3} \vec{p}_{i}}{(2 \pi \hbar)^{3}} \prod_{\vec{k}, \lambda} \frac{d q(\vec{k}, \lambda) d \pi(\vec{k}, \lambda)}{2 \pi \hbar} \exp \left(-\beta H_{\mathrm{C} 1}\right)
$$

with $H_{\mathrm{C} 1}$ given by Eqs. (2.1)-(2.2) and (2.5)-(2.6). Notice that: i) all oscillator coordinates and momenta, for any $(\vec{k}, \lambda)$, are taken at a given $t=0$ (not written explicitly), and ii) the right-hand-side of Eq. (2.7) contains a functional integration over $q(\vec{k}, \lambda)$ and $\pi(\vec{k}, \lambda)$.

Next we change $\vec{p}_{i}-q / c \vec{A}_{\mathrm{Cl}}\left(\vec{x}_{i}, t\right) \rightarrow \vec{p}_{i}$ in $(2.7)$. Then, we perform successive gaussian integrations, first, over all $\vec{p}_{i}$ and, second, over all $q$ 's and $\pi$ 's. Thus, we get

$$
Z_{N, \mathrm{Cl}}=\frac{1}{N !}\left(\frac{m}{2 \pi \beta \hbar^{2}}\right)^{3 N / 2} Z_{\mathrm{FM}, \mathrm{Cl}}(\beta, \hbar) \int \prod_{i=1}^{N} d^{3} \vec{x}_{i} \cdot \exp \left\{-\beta \sum_{i>j=1}^{N} \frac{q^{2}}{4 \pi\left|\vec{x}_{i}-\vec{x}_{j}\right|}\right\} .
$$

where $Z_{\mathrm{EM}, \mathrm{Cl}}$ is the partition function for the classical EM field, namely:

$$
Z_{\mathrm{EM}, \mathrm{Cl}}(\beta, \hbar)=\int \prod_{\vec{k}, \lambda} \frac{d q(\vec{k}, \lambda) d \pi(\vec{k}, \lambda)}{2 \pi \hbar} \exp \left(-\beta H_{\mathrm{EU}, \mathrm{Cl}}\right)=\prod_{\vec{k}}\left(\frac{1}{\omega^{2} \beta^{2} \hbar^{2}}\right) .
$$

Therefore, as far as the partition function is concerned, things occur as if the particles interact only through the instantaneous Coulomb potential while the transverse EM field decouples from the former and behaves as a free field. This fact, to our knowledge, does not seem to have been pointed out before. It also seems to be a direct consequence of the minimal coupling (gauge invariance). Notice that Eq. (2.9) yields the RayleighJeans law. In fact, by taking logarithms in Eq. (2.9) we obtain the energy per unite volume as

$$
\frac{1}{V}\left\langle H_{\mathrm{EM}, \mathrm{Cl}}\right\rangle=-\frac{1}{v} \frac{\partial}{\partial \beta} \ln Z_{\mathrm{E}, \mathrm{M}, \mathrm{Cl}}(\beta, \hbar)=\sum_{\lambda=1,2} \int \frac{d^{3} \vec{k}}{(2 \pi)^{3}} \frac{1}{\beta} .
$$

In turn, by using $\sum_{\lambda=1,2} d^{3} \vec{k}(2 \pi)^{-3}=8 \pi v^{2} c^{-3} d v$ in Eq. (2.10), we get the energy per unit volume between $v$ and $\nu+d v$ as $8 \pi v^{2}\left(c^{3} \beta\right)^{-1}$, which is the Rayleigh-Jeans formula.

Taking the partition function as starting point it is easy to evaluate the classical mean value of any dynamical variable. For instance, since $\vec{A}_{\mathrm{Cl}}(\vec{x}, t)$ is linear in $q(\vec{k}, \lambda)$ we have, by symmetrical integration

$$
\left\langle\vec{A}_{\mathrm{Cl}}(\vec{x})\right\rangle=\frac{1}{Z_{\mathrm{N}, \mathrm{Cl}}} \frac{1}{N !} \int \prod_{c=1}^{N} \frac{d^{3} \vec{x}_{i} d^{3} \vec{p}_{i}}{(2 \pi \hbar)^{3}} \prod_{\vec{k}, \lambda} \frac{d q(\vec{k}, \lambda) d \pi(\vec{k}, \lambda)}{2 \pi \hbar} \vec{A}_{\mathrm{Cl}}(\vec{x}) \exp \left(-\beta H_{\mathrm{Cl}}\right)=0 .
$$

Analogously, for the magnetic field $\vec{B}_{\mathrm{Cl}}=\vec{\nabla} \wedge \vec{A}_{\mathrm{Cl}}$ we get

$$
\left\langle\vec{B}_{\mathrm{Cl}}(\vec{x})\right\rangle=0 \text {. }
$$


For the electric field $\vec{E}=-\left(\hat{c} \vec{A}_{\mathrm{Cl}} / c \partial t\right)+\vec{l} \sum_{i=1}^{N}\left(q / \mathbf{4} \pi\left|\vec{x}-\vec{x}_{i}\right|\right)$ we have $\left\langle\vec{E}_{\mathrm{Cl}}(\vec{x})\right\rangle$
$\vec{V}\left\langle V_{\mathrm{Cl}}(\vec{x})\right\rangle$, with $\left\langle V_{\mathrm{Cl}}(\vec{x})\right\rangle=\frac{1}{Z_{N, \mathrm{Cl}}} \frac{1}{N !} \int \prod_{i=1}^{N} \frac{d^{3} \vec{x}_{i} d^{3} \vec{p}_{i}}{(2 \pi h)^{3}} \prod_{\vec{k}, \lambda} \frac{d q(\vec{k}, \lambda) d \pi(\vec{k}, \lambda)}{2 \pi \hbar} \sum_{i=1}^{N} \frac{q}{4 \pi\left|\vec{x}-\vec{x}_{i}\right|} \exp \left(-\beta H_{\mathrm{Cl}}\right)$,

since in the Coulomb gauge $-\hat{C} \vec{A}_{\mathrm{Cl}} / c \partial t$ is the canonical momentum,

$$
-\frac{1}{c} \frac{\partial A_{\mathrm{Cl}}(\vec{x}, t)}{\partial t}=-i \sum_{\lambda=1,2} \int \frac{d^{3} \vec{k}}{(2 \pi)^{3 / 2}} c(\vec{k}) \vec{\varepsilon}(\vec{k}, \lambda) \pi(\vec{k}, \lambda) e^{i \vec{k} \vec{x}}
$$

and, by symmetrical integration in $x(\vec{k}, \lambda)$ we get

$$
\left\langle\frac{\partial \vec{A}_{\mathrm{Cl}}(\vec{x}, t)}{\partial t}\right)=\frac{\left\langle\partial \vec{A}_{\mathrm{Cl}}(\vec{x})\right\rangle}{\partial t}=0
$$

For any vector $\vec{a}$, by performing an overall translation $\vec{x}_{i} \rightarrow \vec{x}_{i}+\vec{a}$, we get $\left\langle V_{\mathrm{Cl}}(\vec{x}+\vec{a})\right\rangle$ $=\left\langle V_{\mathrm{Cl}}(\vec{x})\right\rangle$, or, equivalently, $\left\langle\boldsymbol{V}_{\mathrm{Cl}}(\vec{x})\right\rangle=$ const., and therefore,

$$
\left\langle\vec{E}_{\mathrm{Cl}}(\vec{x})\right\rangle=0 \text {. }
$$

By a straightforward generalization of the latter arguments it follows that if the background is not homogeneous but a periodic distribution of heavy charges without internal degrees of freedom, the mean value for the electrostatic potential and for the electric. field are periodic with the same periodicity as that of the background.

For the kinetic part $H_{p, \mathrm{Cl}}$ of the hamiltonian, by performing the gaussian integrations, we have

$$
\begin{aligned}
\left\langle H_{p, \mathrm{Cl}}\right\rangle & =\frac{1}{Z_{N, \mathrm{Cl}}} \frac{1}{N !} \int \prod_{i=1}^{x} \frac{d^{3} \vec{x}_{i} d^{3} \vec{p}_{i}}{(2 \pi \hbar)^{3}} \prod_{\vec{k}, 2} \frac{d q(\vec{k}, \lambda) d \pi(\vec{k}, \lambda)}{2 \pi \hbar} H_{p, \mathrm{Cl}} \exp \left(-\beta H_{\mathrm{Cl}}\right) \\
& =\frac{3 N}{2} \frac{1}{\beta}
\end{aligned}
$$

And for the electrostatic energy $H_{\mathrm{C}}$ we get

$$
\left\langle H_{c, \mathrm{c} 1}\right\rangle=\left\{\int \prod_{i=1}^{N} d^{3} \tilde{x}_{i} \exp \left(-\beta H_{c}\right)\right\}^{-1} \int \prod_{i=1}^{N} d^{3} x_{i} H_{c} \exp \left(-\beta H_{c}\right) .
$$

From Eqs. (2.1) - (2.2), (2.10) and (2.14) - (2.15) it follows

$$
\left\langle H_{\mathrm{Cl}}\right\rangle=\frac{3 N}{2} \frac{1}{\beta}+V \sum_{i=1,2} \int \frac{d^{3} \vec{k}}{(2 \pi)^{3}} \frac{1}{\beta}+\left\langle H_{c, \mathrm{Cl}}\right\rangle=-\frac{\partial}{\partial \beta} \ln Z_{N, \mathrm{Cl}} .
$$

\section{Quantum Statistical Mechanics for Non-Relativistic Electrodynamics}

Consider $N$ quantum $(Q)$ identical particles (bosons or fermions) interacting with a quantized EM field $\vec{A}_{Q}(\vec{x})$. The hamiltonian $H_{Q}$ for the system in the Coulonb gauge $\left(\vec{V} \vec{A}_{Q}(\vec{x})=0\right)$ and in the Schrodinger picture is given by

$$
H_{Q}=H_{p, Q}+H_{\mathrm{SM}, Q}+H_{\ell}, \quad H_{p, Q}=\frac{1}{2 m} \sum_{c=1}^{N}\left[\vec{P}_{i}-\frac{q}{c} \vec{A}_{Q}\left(\vec{x}_{i}\right)\right]^{2},
$$




$$
\begin{aligned}
& H_{\mathrm{F}, \mathrm{U}, Q}=\frac{1}{2} \sum_{\lambda=1,2} \int \frac{d^{3} \vec{k}}{(2 \pi)^{3}}|c(\vec{k})|^{2}\left[|\Pi(\vec{k}, \lambda)|^{2}+\omega^{2}|Q(\vec{k}, \lambda)|^{2}\right], \\
& H_{c}=\sum_{i>j=1}^{N} \frac{q^{2}}{4 \pi\left|\vec{x}_{i}-\vec{x}_{j}\right|},
\end{aligned}
$$

where

$$
\vec{A}_{(\ell}(\vec{x})=i c \sum_{\lambda=1,2} \int \frac{d^{3} \vec{k}}{(2 \pi)^{3 / 2}} c(\vec{k}) \vec{\varepsilon}(\vec{k}, \lambda) Q(\vec{k}, \lambda) e^{i \vec{k} \vec{x}}
$$

$Q(\vec{k}, \hat{\lambda})$ and $\Pi(\vec{k}, \lambda)$ are the operators associated to $q(\vec{k}, \lambda, t)$ and $\pi(\vec{k}, \lambda, t)$. They satisfy canonical commutation rules and

$$
Q^{+}(\vec{k}, \lambda)=Q(-\vec{k}, \hat{\lambda}), \quad \Pi^{+}(\vec{k}, \lambda)=\Pi(-\vec{k}, \lambda)
$$

Eq. (3.4) tells us that even if $\vec{A}_{Q}(\vec{x})$ is selfadjoint, $Q(\vec{k}, \lambda)$ and $\Pi(\vec{k}, \lambda)$ are not. In the first Eq. (3.2) we have omitted the energy of the vacuum. Later on, we will come back to this point.

In the subspace of $N$ identical particles in an EM field, the set

$$
\left\{\frac{1}{\sqrt{N !}} \sum_{P}(\mp 1)^{\varepsilon(P)}\left|\vec{x}_{1}(P 1), \ldots, \vec{x}_{N}(P N) ;\{\tilde{q}\}\right\rangle\right\}
$$

forms an orthonormal basis. The sum $\sum_{P}$ is extended to all possible different permutations of $N$ objects. The minus sign is for fermions and the plus one for bosons while $\varepsilon(P)$ is the order of each permutation $P$. The state $\left\{\vec{x}_{1}(P 1), \ldots, \vec{x}_{N}(P N) ;\{\tilde{q}\}\right\rangle$ is that one in which particle 1 is in $\vec{x}_{P_{1}}, \ldots$, particle $N$ in $\vec{x}_{P N}$ and the harmonic oscillators of the EM field are in a state denoted by $\{q\}$. Using this basis, the quantum partition function in the canonical ensemble is given by (compare with [10], section 10.4)

$$
\begin{aligned}
Z_{N, Q}= & \operatorname{Tr}\left[\exp \left(-\beta H_{Q}\right)\right] \\
= & \frac{1}{N !} \int \prod_{i=1}^{N} d^{3} \vec{x}_{i} \prod_{\vec{k}, \lambda} d \tilde{q}(\vec{k}, \lambda) \sum_{P}(\mp 1)^{\varepsilon(P)}\left\langle\vec{x}_{1}, \ldots, \vec{x}_{N} ;\{\tilde{q}\}\right. \\
& \left.\times\left|\exp \left(-\beta H_{Q}\right)\right| \vec{x}_{1}(P 1), \ldots, \vec{x}_{N}(\mathrm{PN}) ;\{\tilde{q}\}\right\rangle .
\end{aligned}
$$

All the matrix elements appearing in Eq. (3.5) are "probability amplitudes" for the system being at $\tau=0$ in $\left|\vec{x}_{1}(P 1), \ldots, \vec{x}_{N}(P N) ;\{\tilde{q}\}\right\rangle$ to be at $\tau=\beta \hbar$ in $\left|\vec{x}_{1}, \ldots, \vec{x}_{N} ;\{\tilde{q}\}\right\rangle$, which can be represented by means of Feynman path integrals as

$$
\begin{aligned}
& \left\langle\vec{x}_{1}, \ldots, \vec{x}_{N} ;\{\tilde{q}\}\left|\exp \left(-\beta H_{Q}\right)\right| \vec{x}_{1}{ }^{\prime}, \ldots, \vec{x}_{N} ;\{\tilde{q}\}\right\rangle \\
& =\int \prod_{i=1}^{N} \prod_{\tau} \frac{d^{3} \vec{x}_{i}(\tau) d^{3} \vec{p}_{i}(\tau)}{(2 \pi \hbar)^{3}} \prod_{\vec{k}, \lambda, \tau} \frac{d q(\vec{k}, \lambda, \tau) d \pi(\vec{k}, \lambda, \tau)}{2 \pi \hbar} \\
& \quad \times \exp \left\{\frac{1}{\hbar} \int_{a}^{\beta h} d \tau\left[i \sum_{j=1}^{N} \vec{p}_{j}(\tau) \dot{\vec{x}}_{j}(\tau)+i \sum_{\lambda=1,2} \int d^{3} \vec{k} \pi(\vec{k}, \lambda, \tau) \dot{q}(\vec{k}, \lambda, \tau)-H_{\mathrm{Cl}}(\tau)\right]\right\},
\end{aligned}
$$


where $H_{\mathrm{cl}}(\tau)$ is given in section 2. Evaluating $\mathrm{Eq}$. (3.6) (see appendix A) and substituting it into (3.5) we get

$$
\begin{aligned}
& Z_{N, Q}=\frac{1}{N !} Z_{\mathrm{E} U, Q}(\beta, \hbar) \int \prod_{i=1}^{N} d^{3} \vec{x}_{i} \sum_{P}(\mp 1)^{\varepsilon(P)} \prod_{j=1}^{N} \int_{\vec{x}_{j}\left(P_{j}\right)}^{\vec{x}_{j}} D^{3} \vec{y}_{j} \exp [G(\vec{y})] \\
& Z_{\mathrm{EM}, Q}(\beta, \hbar)=\prod_{\vec{k}} \frac{1}{2(\cosh \omega \beta \hbar-1)},
\end{aligned}
$$

where we are using the compact notation

$$
D^{3} \vec{y}_{i} \equiv \lim _{M \rightarrow \infty}\left(\frac{m}{2 \pi \hbar \delta}\right)^{3 / 2} \prod_{l=1}^{M} \frac{d^{3} \vec{y}_{i, l}}{\left(m^{-12 \pi \hbar \delta)^{3 / 2}}\right.}
$$

and the exponent $G(\vec{y})$ is given by

$$
\begin{aligned}
G(\vec{y}) \equiv & \frac{1}{\hbar} \int_{0}^{\beta \hbar} d \tau\left[-\frac{m}{2} \sum_{i=1}^{N} \dot{\vec{y}}_{i}^{2}(\tau)-\sum_{i>j=1}^{N} \frac{q^{2}}{4 \pi\left|\vec{y}_{i}(\tau)-\vec{y}_{j}(\tau)\right|}\right] \\
& -\frac{q^{2}}{2 \hbar} \sum_{i, j=1}^{N} \sum_{\lambda=1,2} \int \frac{d^{3} \vec{k}}{(2 \pi)^{3}} \frac{|c(\vec{k})|^{2}}{\omega} \int_{0}^{\beta \hbar} d \tau \int_{0}^{\beta \hbar} d \tau^{\prime}\left[\vec{\varepsilon}(\vec{k}, \lambda) \dot{\vec{y}}_{i}(\tau)\right] \\
& \times \exp \left\{i \vec{k}\left[\vec{y}_{i}(\tau)-\vec{y}_{i}(\tau)\right]\right\} f\left(\tau-\tau^{\prime}, \beta \hbar\right)\left[\vec{\varepsilon}(\vec{k}, \lambda) \dot{\vec{y}}_{j}\left(\tau^{\prime}\right)\right] .
\end{aligned}
$$

The function $f(\tau-\tau, \beta h)$ has the form

$$
f\left(\tau-\tau^{\prime}, \beta h\right)=\frac{e^{-\omega\left|\tau-\tau^{\prime}\right|}+e^{\omega\left|\tau-\tau^{\prime}\right|} e^{\omega \beta \hbar}}{1-e^{-\omega \beta \hbar}} .
$$

Remember that for a partition of the interval $[0, \beta \hbar]$ into $M+1$ subintervals of length $\delta$, and according to the midpoint rule [17], the factor $\exp \left\{\vec{i}\left(\vec{y}_{i}(\tau)-\vec{y}_{j}\left(\tau^{\prime}\right)\right]\right\}$ gives rise to

$$
\exp \left\{\frac{i}{2} \vec{k}\left[\left(\vec{y}_{i, l}+\vec{y}_{i, l-1}\right)-\left(\vec{y}_{j, l^{\prime}}+\vec{y}_{j, l-1}\right)\right]\right\}
$$

The partition function for the EM field $Z_{\mathrm{F}, \mathrm{M}, Q}(\beta, \hbar)$, Eq. (3.8), can be written as

$$
Z_{\mathrm{EM}, \ell}(\beta, \hbar)=\prod_{\vec{k}, \lambda} \frac{e^{-\omega \beta \hbar / 2}}{1-e^{-\omega \beta \hbar}} .
$$

The factor $\exp (-\omega \beta \hbar / 2)$ corresponds to the ground state (vacuum state in a Fock space) of the oscillator associated to $(\vec{k}, \lambda)$. In all what we have done we have ignored the zeropoint energy shift in such a way that instead of the $H_{\mathrm{EM}, Q}$ of Eq. (3.2) we should have written

$$
H_{\mathrm{EM}, Q}-\frac{\hbar}{2} V_{\lambda=1,2} \int \frac{d^{3} \vec{k}}{(2 \pi)^{3}}|c(\vec{k})|^{2} \omega .
$$

This extra term cancels the contribution from the ground state in $Z_{E M, Q}(\beta, \hbar)$ and transforms Eq. (3.12) into

$$
Z_{\mathrm{EM}, Q}(\beta, \hbar)=\prod_{\vec{k}, \lambda} \frac{1}{1-e^{-i \omega \beta}},
$$


which is Planck's partition function. See, for instance, [15] for a study of the partition function for the free quantized EM field.

Notice that Eq. (3.7) is the product of the quantum partition functions for the free EM field and for $N$ particles interacting instantaneously through a Coulomb potential and at different times by means of a "propagator"

$$
\begin{aligned}
D_{i j}^{-1}\left(\vec{y}-\vec{y}^{\prime}, \tau-\tau^{\prime}\right) \equiv & \sum_{\lambda=1,2} \int \frac{d^{3} \vec{k}}{(2 \pi)^{3}} \frac{|c(\vec{k})|^{2}}{\omega} \varepsilon_{i}(\vec{k}, \lambda) \\
& \times \exp \left\{i \vec{k}\left[\vec{y}(\tau)-\vec{y}^{\prime}\left(\tau^{\prime}\right)\right]\right\} f\left(\tau-\tau^{\prime}, \beta \hbar\right) \varepsilon_{j}(k, \lambda) .
\end{aligned}
$$

We also notice that Eq. (3.7) accomplishes FEynmax's elimination of the EM field variables with full generality in the partition function: compare with [10], chapter 9 and 11.

Next, we evaluate the averages values of the quantized operators $\vec{A}_{Q}(\vec{x}), \vec{B}_{Q}(\vec{x}), \vec{E}_{Q}(\vec{x})$ and $H_{Q}$. For any operator 0 we have

$$
\langle 0\rangle=\frac{\operatorname{Tr}\left[\exp \left(-\beta H_{Q}\right) 0\right]}{Z_{N, Q}} .
$$

In the case of $\left\langle\vec{A}_{Q}(\vec{x})\right\rangle$ the matrix elements needed to evaluate the trace are of the type

$$
\begin{aligned}
& \left\langle\vec{x}_{1}, \ldots, \vec{x}_{N} ;\{\tilde{q}\}\left|\exp \left(-\beta H_{Q}\right) Q(\vec{k}, \lambda)\right| \vec{x}_{1}(P 1), \ldots, \vec{x}_{N}(P N) ;\{\tilde{q}\}\right\rangle \\
& =\left\langle\vec{x}_{1}, \ldots, \vec{x}_{N} ;\{\tilde{q}\}\left|\exp \left(-\beta H_{Q}\right)\right| \vec{x}_{1}(P 1), \ldots, \vec{x}_{N}(P N) ;\{\tilde{q}\}\right\rangle \tilde{q}(\vec{k}, \lambda),
\end{aligned}
$$

so, proceeding in the same way as in the partition function, after a gaussian integration, we get

$$
\begin{aligned}
\left\langle\vec{A}_{Q}(\vec{x})\right\rangle= & \frac{Z_{\mathrm{EM}, Q}(\beta, \hbar)}{N ! Z_{N, Q}} \sum_{\lambda=1,2} \int d^{3} \vec{k} \vec{\varepsilon}(\vec{k}, \lambda) e^{i \vec{k} \vec{x}} \int \prod_{i=1}^{N} d^{3} \vec{x}_{i} \sum_{P}(\mp 1)^{\varepsilon(P)} \\
& \times \prod_{j=1}^{N} \int_{\vec{x}_{j}\left(P_{j}\right)}^{\vec{x}_{j}} D^{3} \vec{y}_{j} \frac{(C+D)\left(e^{\omega \beta \hbar}-1\right)}{(\cosh \omega \beta h-1)} e^{G(\vec{y})},
\end{aligned}
$$

with $C$ and $D$ given by

$$
\begin{aligned}
& C=-\frac{1}{2 \omega} \int_{0}^{\beta h} d \tau e^{-\omega \tau} \sum_{i=1}^{N} \gamma_{i}(\tau), \quad D=-\frac{1}{2 \omega} \int_{0}^{\beta h} d \tau e^{-\omega(\beta h-t)} \sum_{i=1}^{N} \gamma_{i}(\tau), \\
& \gamma_{i}(\tau)=q \frac{c(\vec{k})}{(2 \pi)^{3 / 2}} \vec{\varepsilon}(\vec{k}, \lambda) \dot{\vec{y}}_{i}(\tau) e^{i \vec{k} \vec{y}_{i}(\tau)} .
\end{aligned}
$$

Analogously, we get

$$
\begin{aligned}
\left\langle\vec{B}_{Q}(\vec{x})\right\rangle= & \frac{Z_{\mathrm{EM}, Q}(\beta, h)}{N ! Z_{N, Q}} \sum_{i=1,2} \int d^{3} \vec{k} \vec{k} \wedge \vec{\varepsilon}(\vec{k}, \lambda) e^{i \vec{k} \vec{x}} \int \prod_{i=1}^{N} d^{3} \vec{x}_{i} \sum_{P}(\mp 1)^{\varepsilon(P)} \\
& \times \prod_{j=1}^{N} \int_{\vec{x}_{j}\left(P_{j}\right)}^{\vec{x}_{j}} D^{3} \vec{y}_{j} \frac{(C+D)\left(e^{\omega \beta \hbar}-1\right)}{(\cosh \omega \beta \hbar-1)} e^{G(\vec{y})} .
\end{aligned}
$$


The electric field is the sum of two terms,

$$
\left\langle\vec{E}_{Q}(\vec{x})\right\rangle=\left\langle i \sum_{i=1,2} \int \frac{d^{3} \vec{k}}{(2 \pi)^{3 / 2}} c(\vec{k}) \vec{\varepsilon}(\vec{k}, \lambda) \Pi(\vec{k}, \lambda) e^{i \vec{k} \vec{x}}\right\rangle+\left\langle\vec{\Gamma} \sum_{i=1}^{N} \frac{q}{4 \pi\left|\vec{x}-\vec{x}_{i}\right|}\right\rangle .
$$

In the first one we have matrix elements of type

$$
\left\langle\vec{x}_{1}, \ldots, \vec{x}_{N} ;\{\tilde{q}\}\left|\exp \left(-\beta H_{Q}\right) \Pi(\vec{k}, \lambda)\right| \vec{x}_{1}(P 1), \ldots, \vec{x}_{N}(P N) ;\{\tilde{q}\}_{i} \cdot\right.
$$

To evaluate them we first perform the integration over all the $\pi(\vec{k}, \lambda)$, which is gaussian, and get a factor

$$
\int_{0}^{\beta \hbar} d \tau \dot{q}(\vec{k}, \lambda, \tau)
$$

which, by the trace condition $q(\vec{k}, \lambda, \beta h)=q(\vec{k}, \lambda, 0)=\tilde{q}(\vec{k}, \lambda)$, is identically zero. Therefore,

$$
\begin{aligned}
\left\langle\vec{E}_{Q}(\vec{x})\right\rangle= & \frac{Z_{\mathrm{EM}, Q}(\beta, h)}{N ! Z_{N, Q}} \int \prod_{i=1}^{N} d^{3} \vec{x}_{i}\left(\vec{V} \sum_{i=1}^{N} \frac{q}{4 \pi\left|\vec{x}-\vec{x}_{i}\right|}\right) \\
& \times \sum_{P}(\mp 1)^{\varepsilon(P)} \prod_{j=1}^{N} \int_{\vec{x}_{j}\left(P_{j}\right)}^{\vec{x}_{j}} D^{3} \vec{y}_{j} e^{G(\vec{y})} .
\end{aligned}
$$

In the quantum case, and differently from what happens in the classical one, the integration of the EM variables gives rise to an interaction among the particles, bilinear in their velocities and propagated by the function given in Eq. (3.13). Besides, $\left\langle\vec{A}_{Q}(\vec{x})\right\rangle$ and $\left\langle\vec{B}_{Q}(\vec{x})\right\rangle$ contain the sum $C+D$, which is basically a velocity, see Eqs. (3.14)-(3.17).

\section{Classical Limit and Leading Quantum Corrections for the Partition Function}

In this section we shall study what happens with the quantum quantities when the classical limit $h \rightarrow 0$ is taken at fixed non-vanishing temperature $\left(m c^{2} \gg \beta^{-1} \neq 0\right)$. We start with the partition function, Eq. (3.7). To analyse the behaviour of the path integral of (3.7) for $h \rightarrow 0$ we assume that the trajectories $\vec{y}_{i}(\tau)$ can be written in general as

$$
\vec{y}_{i}(\tau)=\vec{x}_{i}(P i)+\frac{\vec{x}_{i}-\vec{x}_{i}(P i)}{\beta \hbar} \tau+\vec{\xi}_{i}(\tau)
$$

with $\vec{\xi}_{i}(\tau)$ satisfying the boundary conditions

$$
\vec{\xi}_{i}(0)=\vec{\xi}_{i}(\beta h)=0 .
$$

We recall (see, for instance, [16], chapter 10) that for $N$ noninteracting identical particles the partition function is given by

$$
Z_{N, Q}^{0}=\frac{1}{N !}\left(\frac{m}{2 \pi \beta \hbar^{2}}\right)^{3 N / 2} \sum_{P}(\mp 1)^{\varepsilon(P)} \int \prod_{i=1}^{N} d^{3} \vec{x}_{i} \exp \left\{-\frac{m}{2 \beta \hbar^{2}} \sum_{i=1}^{N}\left[\vec{x}_{i}-\vec{x}_{i}(P i)\right]^{2}\right\},
$$

so that if we let $\hbar \rightarrow 0$ the only permutation which contributes is the identity $(I)$ :

$$
Z_{N, Q}^{0} \underset{h \rightarrow 0}{\longrightarrow} \frac{1}{N !}\left(\frac{m}{2 \pi \beta \hbar^{2}}\right)^{3 N / 2} \mathrm{~V}^{N}=Z_{N, C 1}^{0}
$$


$r$ being the volume occupied by the particles. Moreover, when the particles interact through an instantaneous local potential, the leading quantum corrections to the free energy of orders $\hbar$ and $\hbar^{2}$ (at fixed $\beta \neq \infty$ ) are also due to the identity. The contributions arising from quantum statistics (say, other permutations different from 1) produce exchange corrections of higher order in $\hbar$ both for non-interacting particles and for particles interacting through instantaneous potentials: their specific form (of order $\hbar^{3}$ ) for the free energy in the case of non-interacting particles is well known. About these matters, see, for instance, chapters 10 and 11 in [16] and section 33 in [18]. This tells us that when we introduce, in addition, the interaction mediated by the dynamical EM field which, for several purposes, could be regarded as a small perturbation, the leading quantum corrections to the partition function (to be summarized neatly by Eq. (4.5), our main result) will also come from the identity permutation. The exchange corrections due to other permutations different from I will be of higher order in $\hbar$ in the present case as well, (see also section 5). Accordingly, in $\mathrm{Eq}$. (3.7) we will take $P=I$, which implies that Eq. (4.1) should be replaced by

$$
\vec{y}_{i}(\tau)=\vec{x}_{i}+\vec{\xi}_{i}(\tau), \quad \vec{\xi}_{i}^{2}(\tau)-\frac{\beta \hbar^{2}}{m} .
$$

The last estimate of the size of $\vec{\xi}_{i}(\tau)$ comes from equaling the kinetic energy to 1 and agrees with the standard philosophy for brownian paths, [17]. Notice that

$$
\dot{\vec{\xi}}_{i}^{2}(\tau) \sim \frac{1}{\beta m}
$$

and that $\tau, \tau^{\prime},\left|\tau-\tau^{\prime}\right| \sim \beta \hbar$. A lengthy evaluation of Eq. (3.7) under the hypothesis (4.3) and (4.4) yields our main result, (for a detailed derivation, see appendix $B$ ):

$$
\begin{aligned}
Z_{N, Q}= & \frac{1}{N !} Z_{\mathrm{E}, \mathrm{II}, Q}(\beta, \hbar)\left(\frac{m}{2 \pi \beta \hbar^{2}}\right)^{3 N / 2} \int \prod_{i=1}^{N} d^{3} \vec{x}_{i} \exp \left\{-\beta \sum_{i>j=1}^{N} \frac{q^{2}}{4 \pi\left|\vec{x}_{i}-\vec{x}_{j}\right|}\right\} \\
& \times\left\{1-2 N \frac{\beta \hbar^{2}}{m^{2}} \ell+2 N \frac{\beta^{2} \hbar^{2} c^{2}}{m} \ell+\frac{3}{2} \frac{\beta^{3} \hbar^{2}}{m}\left(\frac{q^{2}}{2 \pi}\right)^{2} \Delta+O\left(\hbar^{3}\right)\right\},
\end{aligned}
$$

with $\mathscr{C}$ given by

$$
\mathscr{C} \equiv\left(\frac{q}{c}\right)^{2} \int \frac{d^{3} \vec{k}}{(2 \pi)^{3}}|c(\vec{k})|^{2}
$$

For $N=1,(4.5)$ reduces to

$$
Z_{N=1, Q}=Z_{\text {EM,Q }}(\beta, \hbar)\left(\frac{m}{2 \pi \beta \hbar^{2}}\right)^{3 / 2}\left\{1-2 \frac{\beta \hbar^{2}}{m^{2}} \mathscr{C}+2 \frac{\beta^{2} \hbar^{2} c^{2}}{m} \mathscr{C}+O\left(\hbar^{3}\right)\right\} \text {. }
$$

Notice that the last curly bracket is equal to or smaller than 1 since $\beta^{-1} \ll m c^{2}$, in agreement with the bound (A.7) given in appendix $A$.

Eq. (4.5) together with

$$
Z_{\mathrm{EM}, Q}(\beta, \hbar)=\prod_{\vec{k}} \frac{1}{2(\cosh \omega \beta \hbar-1)} \underset{\hbar \rightarrow 0}{\rightarrow} \prod_{\vec{k}} \frac{1}{\omega^{2} \beta^{2} \hbar^{2}}=Z_{\mathrm{EMI}, \mathrm{Cl}}(\beta, \hbar)
$$

implies

$$
Z_{N, Q} \underset{\hbar \rightarrow 0}{\rightarrow} Z_{\Lambda, \mathrm{Cl}},
$$

which is the generalization of theorem 15.9 of [11] to dynamical EM fields. Notice also that Eq. (4.5) gives explicit expressions for the first quantum corrections to the classical 
partition function, and, therefore, to the classical behaviour of $N$ identical charged particles in an EM field.

Next, we study the limit $\hbar \rightarrow 0$ of the average values of $\vec{A}_{Q}, \vec{B}_{Q}$ and $\vec{E}_{Q}$. Proceeding in the same way as for $Z_{N, Q}$ (see appendix $C$ for details) we get

$$
\begin{aligned}
& \left\langle\vec{A}_{Q}(\vec{x})\right\rangle \underset{\hbar \rightarrow 0}{\longrightarrow} 0=\left\langle\vec{A}_{\mathrm{Cl}}(\vec{x})\right\rangle, \\
& \left\langle\vec{B}_{Q}(\vec{x})\right\rangle \underset{\hbar \rightarrow 0}{\longrightarrow} 0=\left\langle\vec{B}_{\mathrm{Cl}}(\vec{x})\right\rangle, \\
& \left\langle\vec{E}_{Q}(\vec{x})\right\rangle \underset{\hbar \rightarrow 0}{\longrightarrow} 0=\left\langle\vec{E}_{\mathrm{Cl}}(\vec{x})\right\rangle .
\end{aligned}
$$

For the hamiltonian we have, that by differentiating logarithmically with respect to $\beta$ and taking the limit $h \rightarrow 0$ :

$$
\left\langle H_{Q}\right\rangle \underset{\hbar \rightarrow 0}{\longrightarrow}\left\langle H_{\mathrm{Cl}}\right\rangle \text {. }
$$

Eqs. (4.8)-(4.12) tell us that when the limit $h \rightarrow 0$ is taken the classical results are recovered.

Before finishing this section same remarks are in order.

Remark 1. The method used here, based heavily upon Feynman's path integrals, seems far more advantageous for the present problem than the one inspired in the semiclassical approximation of WIGNER-KIRKwood [19], where one would be led to solve some sort of Bloch equation by approximate methods. Moreover, our study is in the line of the work of Feynnian [9], where he recovered the Schrödinger equation from the path integral formalism by studying the behaviour of the integral for small times. The validity of Eq. (4.8), the fact that without the EM field interaction the quantum fermion system with pure Coulomb forces coincides with the classical Coulomb system as $\hbar \rightarrow 0$, and the proof of stability of fermion matter for pure Coulomb forces by LIEB and THIRRING [20] indicates that, at least, in the classical limit, stability of matter should also hold for the system described by Eq. (3.7).

Remark 2. Eqs. (4.5) and (4.6) seem to imply that when the cut-off function $c(\vec{k})$ is removed (namely, one would set $c(\vec{k})=1$ for $|\vec{k}| \leqq k_{\max }, c(\vec{k})=0$ for $|\vec{k}|>k_{\max }$, with $\left.k_{\max } \rightarrow+\infty\right)$, one would find unavoidably a cubic ultraviolet divergence $\left(\mathscr{C}=(q / c)^{2}\right.$ $\left.\times\left(k_{\max }^{3} / 6 \pi^{2}\right)\right)$ in the leading quantum corrections to the parition function, even for $N=1$. Such a cubic divergence would be higher than the linear ultraviolet divergence which appears when studying virtual-photon corrections to the electron self-mass in nonrelativistic Quantum Electrodynamics at zero temperature (see, for instance, section $15 \mathrm{~b}$ in [21]).

It turns out that the cubic ultraviolet divergence is superfluous, as the following discussion will show. In fact, the hamiltonian (3.1)-(3.3) for non-relativistic Quantum Electrodynamics and the partition function (3.5) are physically meaningful provided that: i) $\hbar k_{\max } \ll m c$, ii) $\beta^{-1} \ll m c^{2}$. Moreover, the calculation of the quantum corrections to the partition function carried out in this section has physical sense at suitably high temperatures. All these suggest that $\beta^{-1}$ could vary in some interval $\left|\left(\hbar k_{\max }\right)^{2} / 2 m\right|-$ $\varepsilon_{1}<\beta^{-1}<\left|\left(\hbar k_{\max }\right)^{2} / 2 m\right|+\varepsilon_{2}$, where $\varepsilon_{1}$ should be small compared to $\left(\hbar k_{\max }\right)^{2} / 2 m$ and $\varepsilon_{2}$ should be such that the above condition ii) be fulfilled.

For a quick estimate, let $\beta^{-1}$ be of order $\left(h k_{\max }\right)^{2} / 2 m$, which is consistent with both i) and ii). Then, the two leading quantum corrections in Eq. (4.5) become

$$
\frac{\beta \hbar^{2}}{m} \mathscr{C} \simeq \frac{1}{3 \pi^{2}} \frac{q^{2}}{\hbar c} \frac{\hbar k_{\max } c}{m c^{2}}, \quad \frac{\beta^{2} \hbar^{2} c^{2}}{m} \mathscr{C} \simeq \frac{\beta m c^{2}}{3 \pi^{2}} \frac{q^{2}}{\hbar c} \frac{\hbar k_{\max } c}{m c^{2}}
$$


that is, the cubic ultraviolet divergence has disappeared and become linear. One arrives, clearly, at the same conclusion if $\beta^{-1}$ varies in the range $\left|\left(\hbar k_{\max }\right)^{2} / 2 m\right|-\varepsilon_{1}<\beta^{-1}$ $<\left|\left(\hbar k_{\max }\right)^{2} / 2 m\right|+\varepsilon_{2}$. In a forthcoming paper [22] this linear divergence is removed using Quantum Field Theory techniques.

Remark 3. Let us describe the problem in a simple way using its characteristic lengths. The first one is the inteparticle distance $d$ related to the density through $\varrho=d^{-3}$. Other three characteristic lengths are the thermal $\lambda_{\text {th }}$, de Broglie $\lambda_{B}$ and Compton $\lambda_{C}$ wavelengths, given by

$$
\lambda_{\mathrm{th}}=\left(\frac{m}{2 \pi \beta \hbar^{2}}\right)^{-1 / 2}, \quad \lambda_{B}=\frac{\hbar}{(2 m E)^{1 / 2}}, \quad \lambda_{c}=\frac{h}{m c} .
$$

For the actual non-relativistic system (recall conditions i) and ii) of remark 2) the following relation holds : $\lambda_{\text {th }} \geqslant \lambda_{C}$. Moreover, the wavelength $\lambda$ of a photon is such that $2 \pi / \lambda$ $=|\vec{k}|<k_{\max }$, so $\lambda \gg \lambda_{C}$. The condition for the classical limit (validity of the equipartition principle) amounts to $\lambda_{\text {th }} \sim \lambda_{B}$. Notice that for the special case $\lambda \sim \lambda_{\text {th }} \sim \lambda_{B},|\vec{k}| \sim 2 \pi / \hbar$ $\times(m / \beta)^{1 / 2}$, that implies $\hbar|\vec{k}| / m c \sim\left(\beta m c^{2}\right)^{-1 / 2} \ll 1$, which is consistent (with condition i)).

When restricting ourselves to the identity permutation in the analysis of the classical limit we are using implicitly $d \gg \lambda_{\text {th }}$.

For completeness, one could add a fifth length, the Debye wavelength $\lambda_{D}=\left(q^{2} \varrho \beta\right)^{-1 / 2}$ $\gg d$. For a detailed discussion see [13-14].

Remark 4. It is interesting to point out the analogies and differences between the problem studied here and the polaron one, (one electron interacting with a scalar phonon field) as studied by Feynman [15]. There, the path integral formalism is also used, but restricted to very large $\beta$, which means replacing Eq. (3.11) by $\exp \left(-\omega\left|\tau-\tau^{\prime}\right|\right)$. Notice that in Eq. (3.11) $\beta$ appears multipled by $\hbar$, so, making the approximation $\beta \rightarrow \infty$ in Eq. (3.11) would be equivalent in some way to $\hbar \rightarrow \infty$, which differs from what we have done $(\hbar \rightarrow 0)$. There are also some other differences due to the vectorial nature of the EM field, the minimal coupling, the number of particles, etc.

\section{Discussion of Other Methods: The Quasiconstant Field Approximation}

In this section we are going to recover the classical result for the partition function by using a different method, which may well be called the quasiconstant field approximation (compare with Halpern and Siegel [5]). We shall also discuss the advantages and disadvantages of this method.

The starting point is the partition function (3.5). Now, to evaluate the propagator (3.6) we divide the $\vec{x}$-space into small cells $\alpha$ of volume $V_{\alpha}$, and in each of them we expand the EM field into a power series around the center $\vec{x}_{\alpha}$ of the cell:

$$
\vec{A}_{\alpha}(\vec{x})=\vec{A}\left(\vec{x}_{\alpha}\right)+\left(x-x_{\alpha}\right)^{i}\left[\partial_{i} \vec{A}(\vec{x})\right]_{\vec{x}_{\alpha}}+\cdots .
$$

By considering only the first term in (5.1) (quasiconstant field approximation), taking into account that in this approximation $\vec{P}$ and $\vec{A}_{\alpha}(\vec{x})$ conmute, proceeding in the same way as in section 3 and taking the limit $V_{\alpha} \rightarrow 0$ we get

$$
\begin{aligned}
Z_{N, Q}^{(0)}= & Z_{\mathrm{EM}, Q}(\beta, \hbar) \frac{1}{N !}\left(\frac{m}{2 \pi \beta \hbar^{2}}\right)^{3 N / 2} \sum_{P}(\mp 1)^{\varepsilon(P)} \int \prod_{i=1}^{N} d^{3} \vec{x}_{i} \\
& \times \exp \left\{-\beta \sum_{i>j=1}^{N} \frac{q^{2}}{4 \pi \mid \vec{x}_{i}\left(p_{i}\right)-\vec{x}_{j}\left(p_{j}\right)}-\frac{m}{2 \beta \hbar^{2}} \sum_{i=1}^{N}\left[\vec{x}_{i}-\vec{x}_{i}(P i)\right]^{2}\right\},
\end{aligned}
$$


where the subscript (0) refers to the approximation adopted in (5.1). In the limit $h \rightarrow 0$ only the identity permutation contributes to $Z_{N, Q}^{(0)}$, so $Z_{N, Q} \rightarrow Z_{N, C l}$ as $h \rightarrow 0$. The other permutations approach zero faster than the quantum correction for $P=I$ derived in section 4 . This agrees with the discussion at the beginning of section 4 .

The same result would have been obtained if instead of the canonical ensemble, one uses the grand canonical one, and describe the particles by Schrödinger (Bose) fields with antiperiodic (periodic) boundary conditions.

The advantage of this method is that it keeps memory of the different permutations. On the other hand, and due to the partition of the $\vec{x}$-space into cells and the expansion for $\vec{A}$ in each cell, the interactions among particles mediated by $\vec{A}$ are restricted to take place within the same cell and not among different ones, while in the method of section 4 , $\vec{A}$ could propagate the interaction among arbitrary points in the $\vec{x}$-space. This feature, together with the fact that in the limit $\hbar \rightarrow 0$ permutations different from the identity tend to zero faster than the quantum corrections for $P=I$, suggest that the method of section 4 is more suitable for evaluating the leading quantum corrections.

\section{Conclusions}

The important result obtained in section 2 is that in Classical Statistics the dynamical or transverse EM degrees of freedom become decoupled from those of the particles in the partition function. This decoupling between matter and radiation implies that the classical average values for $\vec{A}_{\mathrm{Cl}}, \vec{B}_{\mathrm{Cl}}$ and $\vec{E}_{\mathrm{Cl}}$ vanish. Such a decoupling does not take place quantum-mechanically, but it is proved that $Z_{N, Q} \rightarrow Z_{N, \mathrm{Cl}}$ as $\hbar \rightarrow 0$, which generalizes theorem 15.9 of [11], in which the same property is formulated for an external EM field without dynamical degrees of freedom. Explicit expressions for the leading quantum correction to the classical partition function are given, which constitute the main result of the paper.

Appendix A. Evaluation of Eq. (3.5) and a Bound to Eq. (3.6)

In this appendix we will evaluate the matrix element (3.6) which appears in the partition function (3.5). Eq. (3.6) is a path integral over $\vec{x}_{i}(\tau), \vec{p}_{i}(\tau)$ and $q(\vec{k}, \lambda, \tau), \pi(\vec{k}, \lambda, \tau)$ for $i=1, \ldots, N$ and for all $(\vec{k}, \lambda)$. Making a partition of the time interval $[0, \beta \hbar]$ into $M+1$ subintervals of length $\delta$,

$$
\delta=\frac{\beta \hbar}{M+1}
$$

we have for Eq. (3.6)

$$
\begin{aligned}
& \left\langle\vec{x}_{1}, \ldots, \vec{x}_{N} ;\{\tilde{q}\}\left|\exp \left(-\beta H_{Q}\right)\right| \vec{x}_{1}{ }^{\prime}, \ldots, \vec{x}_{N}{ }^{\prime} ;\{\tilde{q}\}\right\rangle \\
& =\int \lim _{M \rightarrow \infty} \prod_{l=1}^{M} \prod_{l=1}^{M+1} \prod_{i=1}^{N} \frac{d^{3} \vec{x}_{i, l} d^{3} \vec{p}_{i, l^{\prime}}}{(2 \pi \hbar)^{3}} \prod_{\vec{k}, \lambda} \frac{d q_{1} d \pi_{l^{\prime}}}{2 \pi \hbar} \\
& \quad \times \exp \left\{\frac { i } { \hbar } \sum _ { l = 0 } ^ { M + 1 } \left[\sum_{i=1}^{N} \vec{p}_{i, l}\left(\vec{x}_{i, l}-\vec{x}_{i, l-1}\right)+\int d^{3} \vec{k} \pi_{l}\left(q_{l}-q_{l-1}\right)\right.\right. \\
& \quad-\delta \sum_{j=1}^{N} \frac{1}{2 m}\left[\vec{p}_{j, l}-i q \sum_{\lambda=1,2} \int \frac{d^{3} \vec{k}}{(2 \pi)^{3 / 2}} c(\vec{k}) \vec{\varepsilon}(\vec{k}, \hat{\lambda}) q_{l} \exp \left[\frac{i}{2} \vec{k}\left(\vec{x}_{j, l}+\vec{x}_{j, l-1}\right)\right]\right]^{2} \\
& \left.\left.\quad-\frac{\delta}{2} \int \frac{d^{3} \vec{k}}{(2 \pi)^{3}}|c(\vec{k})|^{2}\left(\left|\pi_{l}\right|^{2}+\omega^{2} q_{l_{i}}\right)^{\prime}-\delta \sum_{i>j=1}^{N} \frac{q^{2}}{4 \pi\left|\vec{x}_{i, l}-\vec{x}_{j, l}\right|}\right]\right\}, \quad \text { (A.2) }
\end{aligned}
$$


with boundary conditions

$$
\vec{x}_{i, M+1}=\vec{x}_{i}(\beta h)=\vec{x}_{i}, \quad \vec{x}_{i, 0}=\vec{x}_{i}(0)=\vec{x}_{i}{ }^{\prime} ; \quad q_{M+1}=q(\beta \hbar)=\tilde{q}, \quad q_{0}=q(0)=\tilde{q} .
$$

Notice that in Eq. (A.2) we have solved the well-known path integral ambiguity in the coupling of matter to vector potentials by choosing the midpoint $1 / 2\left(\vec{x}_{i, l}+\vec{x}_{i, l-1}\right)$ as argument of the EM field in the product $\vec{x}_{i} \vec{A}\left(\vec{x}_{j}, t\right)[9,17]$. For a lucid discussion of its relationship to the $I$ to ambiguity see also [17].

Each of the $\vec{p}_{i, l}$ and $\pi_{l}$ integrations are gaussian and can be done immediately

$$
\begin{aligned}
& \left\langle\vec{x}_{1}, \ldots, \vec{x}_{N} ;\{\tilde{q}\}\left|\exp \left(-\beta H_{Q}\right)\right| \vec{x}_{1}{ }^{\prime}, \ldots, \vec{x}_{N} ;\{\tilde{\boldsymbol{q}}\}\right\rangle \\
& =\int \lim _{M \rightarrow \infty} \prod_{l=0}^{M+1} \prod_{i=1}^{N} \frac{d^{3} \vec{x}_{i, l}}{\left(m^{-1} 2 \pi \hbar \delta\right)^{3 / 2}} \prod_{\vec{k}, \lambda} \frac{d q_{l}}{(2 \pi h \delta)^{1 / 2}} \exp \left\{\frac { 1 } { \hbar } \sum _ { l = 0 } ^ { M + 1 } \left[-\frac{m}{2 \delta} \sum_{i=1}^{N}\left(\vec{x}_{i, l}-\vec{x}_{i, l-1}\right)^{2}\right.\right. \\
& \quad-\frac{\delta}{2} \sum_{i=1,2} \int \frac{d^{3} \vec{k}}{(2 \pi)^{3}}|c(\vec{k})|^{2}\left(\left|\frac{q_{l}-q_{l-1}}{\delta}\right|^{2}-\omega^{2}\left|q_{l}\right|^{2}\right)-\sum_{i>j=1}^{N} \frac{q^{2}}{4 \pi\left|\vec{x}_{i, l}-\vec{x}_{i, l}\right|} \\
& \left.\left.\quad-q \sum_{i=1}^{N}\left(\vec{x}_{i, l}-\vec{x}_{i, l-1}\right) \sum_{i=1,2} \int \frac{d^{3} \vec{k}}{(2 \pi)^{3 / 2}} c(\vec{k}) \vec{\varepsilon}(\vec{k}, \lambda) q_{l} \exp \left[\frac{i}{2} \vec{k}\left(\vec{x}_{i, l}+\vec{x}_{i, l-1}\right)\right]\right]\right\} .
\end{aligned}
$$

We shall simplify Eq. (A.3) by recalling Feynmax's result [15]:

$$
\begin{aligned}
& \int_{x(0)=x}^{x(U)=x^{\prime}} \lim _{M \rightarrow \infty}\left(\frac{m}{2 \pi \hbar \delta}\right)^{1 / 2} \prod_{l=1}^{M} \frac{d x_{l}}{\left(m^{-12 \pi \hbar \delta)^{1 / 2}}\right.} \\
& \quad \times \exp \left\{-\frac{1}{\hbar} \sum_{t=0}^{M+1} \delta\left[\frac{m}{2}\left(\frac{x_{l}-x_{l-1}}{\delta}\right)^{2}+\frac{m w^{2}}{2} x_{l}^{2}+i x_{l} \gamma_{l}\right]\right\} \\
& =\left(\frac{m \omega}{2 \pi \hbar \sinh \omega U}\right) \exp \left\{-\frac{1}{4 m \omega \hbar} \int_{0}^{U} d t \int_{0}^{U} d t^{\prime} e^{-\omega\left|t-t^{\prime}\right|} \gamma(t) \gamma\left(t^{\prime}\right)-\frac{m \omega}{2 \hbar \sinh \omega U}\right. \\
& \quad \times\left[\left(x^{2}+x^{\prime 2}\right) \cosh \omega U-2 x x^{\prime}+2 A\left(x e \omega U-x^{\prime}\right)+2 B\left(x^{\prime} e^{\omega U}-x\right)\right. \\
& \left.\left.\quad+\left(A^{2}+B^{2}\right) e^{\omega U}-2 A B\right]\right\}
\end{aligned}
$$

with

$$
A=\frac{i}{2 m(j)} \int_{0}^{l} d t e^{-\omega t} \gamma(t), \quad B=\frac{i}{2 m \omega} \int_{0}^{U} d t e^{-\omega(U-t)} \gamma(t) .
$$

Using Eqs. (A.4) and (A.5) to evaluate the integrals over all $q_{l}$ 's in Eq. (A.3), introducing the result into Eq. (3.5) and performing the integration over $\tilde{q}(\vec{k}, \lambda)$, which is also gaussian, we get Eq. (3.7).

Before concluding this section there are some remarks to do about Eq. (3.9).

Remark 1. The exponent in the integrand of (A.3) is the sum of the kinetic energy for the particles (first term), the energy for the free EM field (second term), the Coulomb energy (third term), and the fourth term, which is associated to the interaction between the field and the particles. The last term is purely imaginary, so its exponential has modulus 1. It follows that the propagator (A.3) is bounded by the product of the propagators 
for $\boldsymbol{N}$ quantum particles interacting via a Coulomb potential and for the froe EM field:

$$
\begin{aligned}
& \left|\left\langle\vec{x}_{1}, \ldots, \vec{x}_{N} ;\{\bar{q}\} \mid \exp \left(-\beta H_{Q}\right): \vec{x}_{1}{ }^{\prime}, \ldots, \vec{x}_{N}{ }^{\prime} ;\{\bar{q}\}\right\rangle\right| \\
& \leqq\left\langle\vec{x}_{1}, \ldots, \vec{x}_{N}\left|\exp \left[-\beta\left(H_{p, Q}^{0}+H_{C}\right)\right]\right| \vec{x}_{1}, \ldots, \vec{x}_{N}\right\rangle\left\langle\{\bar{q}\} ; \exp \left(-\beta H_{\mathrm{E}, Q, Q}\right) \mid\{\tilde{q}\}\right\rangle,
\end{aligned}
$$

with $H_{p . Q}^{0}=\sum_{i=1}^{N} \vec{P}_{i}^{2} / 2 m$, which is the generalization of theorem 15.6 of [11] to dynamical
EM fields.

Remark 2. For only one particle $(N=1)$ Eq. (A.6) gives the following bound for the partition function:

$$
Z_{N=1.0} \leq V\left(\frac{m}{2 \pi \beta h^{2}}\right)^{3 / 2} Z_{\text {F.M.Q. }}(\beta, h)
$$

If we introduce the system with only one particle in an external potential $\varphi(\vec{x})$ we have

$$
Z_{N=1.0} \leqq\left(\frac{m}{2 \pi \beta h^{2}}\right)^{3 / 2} Z_{\mathrm{EM}, 0}(\beta, h) \int d^{3} \vec{x} \exp [-\beta \varphi(\vec{x})],
$$

which is the generalization of theorem 9.2 of [11]. For this case, and calling $E_{0}$ the energy of the groun state, we get the bounds

$$
\exp \left(-\beta E_{0}\right) \leqq \operatorname{Tr}\left[\exp \left(-\beta H_{0}\right)\right] \leqq\left(\frac{m}{2 \pi \beta h^{2}}\right)^{3 / 2} Z_{\mathrm{EM}, 0}(\beta, h) \int d^{3} \vec{x} \exp [-\beta \varphi(x)]
$$

Appendix B : Evaluation of (3.7) for $K \rightarrow 0(P=I)$

The derivation of Eq. (4.5) proceeds through several steps: 1) Expansion of $G(\vec{y})$ into a power series in $\hbar$, regarding the series formed by all terms of orders $\hbar^{n}$ with $n \geqq 1$ as a perturbation, 2) Expansion of the exponential of such a perturbation into a power series, 3) Study of the order in $h$ of the different terms obtained in the last expansion and selection of the leading quantum correction, 4) Explicit evaluation of these leading quantum corrections.

Step 1. According to what has been said in section 4, in the limit $\hbar \rightarrow 0$, the leading contribution comes from the identity permutation $P=I$, for which Eqs. (4.2)-(4.4), together with the fact that $\tau, \tau^{\prime},\left|\tau-\tau^{\prime}\right| \sim \beta h$, hold. From these it follows that Eq. (3.10) can be written as

$$
\begin{aligned}
& G(\vec{y})=\frac{m}{2 \hbar} \sum_{i=1}^{N} \int_{0}^{A h} d \tau{\overrightarrow{\xi_{i}}}^{2}(\tau)-\frac{1}{h} \sum_{i>j=1}^{N} \frac{q^{2}}{4 \pi\left|\vec{x}_{i}-\vec{x}_{j}\right|} \\
& \times \int_{0}^{\beta \Lambda} d \tau\left\{1+\left[\xi_{i}(\tau)-\xi_{j}(\tau)\right]_{a} D_{i j}^{\alpha}+\left[\xi_{i}(\tau)-\xi_{j}(\tau)\right]_{x} Q_{i j}^{\alpha \gamma}\left[\xi_{i}(\tau)-\xi_{j}(\tau)\right]_{y}+\cdots\right\} \\
& -\frac{q^{2}}{2 \beta \hbar^{2}} \sum_{i, j=1}^{N} \sum_{l=1,2} \int \frac{d^{3} \vec{k}}{(2 \pi)^{3}} \frac{\mid c(\vec{k})^{2}}{\omega^{2}} \int_{0}^{\beta \hbar} d \tau \int_{0}^{\beta \hbar} d \tau\left[\dot{\varepsilon}(\vec{k}, \lambda) \dot{\xi}_{i}(\tau)\right] \\
& \times e^{i \vec{k}^{\left(\vec{z}_{i}-\vec{z}_{j}\right)}}\left\{1+i \vec{k}\left[\vec{\xi}_{i}(\tau)-\vec{\xi}_{j}\left(\tau^{\prime}\right)\right]-\frac{1}{2}\left[\vec{k}\left(\vec{\xi}_{i}(\tau)-\vec{\xi}_{j}\left(\tau^{\prime}\right)\right]^{2}+\cdots\right\}\right. \\
& \times\left[\vec{\varepsilon}(\vec{k}, \lambda) \dot{\vec{\xi}}_{j}\left(\tau^{\prime}\right)\right]\left\{2+\omega^{2}\left[\frac{1}{12}(\beta h)^{2}+\left|\tau-\tau^{\prime}\right|^{2}-\beta h^{\prime} \tau-\tau^{\prime} \mid\right]\right. \\
& \left.-\frac{1}{24}(\omega \beta \hbar)^{3}+O\left((\beta h)^{4}\right)\right\} \text {, }
\end{aligned}
$$


where the last curly bracket comes from the expansion of $f\left(\tau-\tau^{\prime}, \beta \hbar\right)$ into suitable powers and $D_{i j}^{\alpha}$ and $Q_{i j}^{\alpha}$ are the following dipole-moment and quadrupole-tensor contributions:

$$
\begin{aligned}
& D_{i j}^{x}=\frac{\left(x_{i}-x_{j}\right)^{\alpha}}{\left|\vec{x}_{i}-\vec{x}_{j}\right|^{2}}, \quad Q_{i j}^{\alpha \gamma}=\frac{3\left(x_{i}-x_{j}\right)^{\alpha}\left(x_{i}-x_{j}\right)^{\gamma}-\left(\vec{x}_{i}-\vec{x}_{j}\right)^{2} \delta^{\alpha \gamma}}{\left|\vec{x}_{i}-\vec{x}_{j}\right|^{4}}, \\
& (\alpha, \gamma=1,2,3) .
\end{aligned}
$$

The standard summation conventions for the dummy indices $\alpha, \beta$ have been used in Eq. (B.1).

Next we study the different terms appearing in Eq. (B.1). The free term (kinetic energy) and the electrostatic energy are of order $\hbar^{0}$

$$
G_{0}(\vec{\xi})=G^{0}(\vec{\xi})-\beta H_{c}, \quad G^{0}(\vec{\xi})=-\frac{m}{2 h} \sum_{i=1}^{N} \int_{0}^{\beta \hbar} d \tau \dot{\vec{\xi}}_{i}^{2}(\tau),
$$

with $H_{c}$ given by Eq. (3.2). There is also a term of order $\hbar^{0}$ arising from the particle-field interaction, which vanishes by virtue of the boundary condition (4.2), namely

$$
-\frac{q^{2}}{\beta \hbar^{2}} \sum_{\lambda=1,2} \int \frac{d^{3} \vec{k}}{(2 \pi)^{3}} \frac{|c(\vec{k})|^{2}}{\omega^{2}}\left|\sum_{i=1}^{N} e^{i \vec{k} \vec{x}_{i}} \int_{0}^{\beta \hbar} d \tau \vec{\varepsilon}(\vec{k}, \lambda) \dot{\vec{\xi}}_{i}(\tau)\right|^{2}=0 .
$$

The term linear in $\hbar$ is

$$
G_{1}{ }^{0}(\vec{\xi})=-\frac{q^{2}}{2 \pi \hbar} \sum_{i>j=1}^{N} \frac{D_{i j}^{\alpha}}{\left|\vec{x}_{i}-\vec{x}_{j}\right|} \int_{0}^{\beta \hbar} d \tau \xi_{i, \alpha}(\tau)
$$

since the other possible contribution

vanishes,

$$
\begin{aligned}
G_{1}{ }^{\prime}(\vec{\xi})= & -\frac{i q^{2}}{\beta \hbar^{2}} \sum_{i, j=1}^{N} \sum_{i=1,2} \int_{\lambda^{3}} \frac{d^{3} \vec{k}}{(2 \pi)^{3}} \frac{|c(\vec{k})|^{2}}{\omega^{2}} \\
& \times e^{i \vec{k}\left(\vec{x}_{i}-\vec{x}_{j}\right)} \int_{0}^{\beta \hbar} d \tau \int_{0}^{\beta \hbar} d \tau^{\prime}\left[\vec{\varepsilon}(\vec{k}, \lambda) \overrightarrow{\vec{\xi}}_{i}(\tau)\right]\left\{\vec{k}\left[\vec{\xi}_{i}(\tau)-\vec{\xi}_{j}\left(\tau^{\prime}\right)\right]\right\}\left[\vec{\varepsilon}\left(\vec{k}, \lambda_{i} \dot{\vec{\xi}}_{j}\left(\tau^{\prime}\right)\right]\right.
\end{aligned}
$$

$$
G_{1} F(\vec{\xi})=0
$$

This can be seen by making in its right-hand side the change $\tau \rightarrow \tau^{\prime}$ (or $i \rightarrow j$ ), or by using the boundary condition (4.2). The term of order $h^{2}$ is

$$
G_{2}(\vec{\xi})=G_{2}^{Q}(\vec{\xi})+G_{2}{ }^{F}(\vec{\xi})+G_{2}{ }^{F 2}(\vec{\xi})
$$

where

$$
\begin{aligned}
G_{2} Q(\vec{\xi})= & -\frac{q^{2}}{2 \pi \hbar} \sum_{i>j=1}^{N} \frac{Q_{i j}^{\alpha \gamma}}{\left|\vec{x}_{i}-\vec{x}_{j}\right|} \int_{0}^{\beta \hbar} d \tau \xi_{i, \alpha}(\tau)\left[\xi_{i}(\tau)-\xi_{j}(\tau)\right]_{\gamma}, \\
G_{2}{ }^{F 1}(\vec{\xi})= & \frac{q^{2}}{2 \beta \hbar^{2}} \sum_{i, j=1}^{N} \sum_{\lambda=1,2} \int \frac{d^{3} \vec{k}}{(2 \pi)^{3}} \frac{|c(\vec{k})|^{2}}{\omega^{2}} e^{i \vec{k}\left(\vec{x}_{i}-\vec{x}_{j}\right)} \int_{0}^{\beta \hbar} d \tau \int_{0}^{\beta \hbar} d \tau^{\prime} \\
& \times\left[\vec{\varepsilon}(\vec{k}, \lambda) \dot{\overrightarrow{\xi_{i}}}(\tau)\right]\left\{\vec{k}\left[\vec{\xi}_{i}(\tau)-\vec{\xi}_{j}\left(\tau^{\prime}\right)\right]\right\}^{2}\left[\vec{\varepsilon}(\vec{k}, \lambda) \dot{\left.\overrightarrow{\xi_{j}}\left(\tau^{\prime}\right)\right],}\right.
\end{aligned}
$$




$$
\begin{aligned}
G_{2}{ }^{2}(\vec{\xi})= & -\frac{q^{2}}{2 \beta \hbar^{2}} \sum_{i, j=1}^{N} \sum_{\lambda=1,2} \int \frac{d^{3} \vec{k}}{(2 \pi)^{3}}|c(\vec{k})|^{2} e^{i \vec{k}\left(\vec{x}_{t}-\vec{x}_{j}\right)} \int_{0}^{\beta \hbar} d \tau \int_{0}^{\beta \hbar} d \tau^{\prime}\left[\vec{\varepsilon}(\vec{k}, \lambda) \dot{\vec{\xi}}_{i}(\tau)\right] \\
& \times\left[\frac{1}{12}(\beta \hbar)^{2}+\left|\tau-\tau^{\prime}\right|^{2}-\beta \hbar\left|\tau-\tau^{\prime}\right|\right]\left[\vec{\varepsilon}(\vec{k}, \lambda) \dot{\vec{\xi}}_{j}\left(\tau^{\prime}\right)\right]
\end{aligned}
$$

Upon expanding the square and using Eq. (4.2), (B.8) simplifies to

$$
G_{2}{ }^{F 1}(\vec{\xi})=-\frac{q^{2}}{\beta \hbar^{2}} \sum_{\lambda=1,2} \int \frac{d^{3} \vec{k}}{(2 \pi)^{3}} \frac{|c(\vec{k})|^{2}}{\omega^{2}}\left|\sum_{i=1}^{N} e^{i \vec{k} \vec{x}_{i}} \int_{0}^{\beta \hbar} d \tau\left[\vec{\varepsilon}(\vec{k}, \lambda) \dot{\vec{\xi}}_{i}(\tau)\right]\left[\vec{k} \vec{\xi}_{i}(\tau)\right]\right|^{2}
$$

Regarding $G_{2}{ }^{F 2}(\vec{\xi})$, an integration by parts and the use of Eq. (4.2) lead to

$$
G_{2} F 2(\vec{\xi})=\frac{q^{2}}{\beta \hbar^{2}} \sum_{\lambda=1,2} \int \frac{d^{3} \vec{k}}{(2 \pi)^{3}}|c(\vec{k})|^{2}\left|\sum_{i=1}^{N} e^{i \vec{k} \vec{x}_{i}} \int_{0}^{\beta \hbar} d \tau \vec{\varepsilon}(\vec{k}, \lambda) \vec{\xi}_{i}(\tau)\right|^{2}
$$

Summarizing,

$$
G=-\beta H_{c}+G^{0}+G_{1}{ }^{D}+G_{2}^{Q}+G_{2}{ }^{F 1}+G_{2}{ }^{22}+O\left(|\vec{\xi}|^{3}, \hbar^{3}\right),
$$

where superindices $0, D, Q$ and $F$ stand for free part dipole, quadrupole and contributions arising from the field. Notice that all the terms included in $O\left(|\vec{\xi}|^{3}, h^{3}\right)$ are, at least, cubic in $\vec{\xi}$ and $\hbar$.

Step 2. We consider $G_{1}{ }^{D}+G_{2}+G_{2}{ }^{F 1}+G_{2}{ }^{22}+O\left(|\vec{\xi}|^{3}, h^{3}\right)$ as a perturbation to $G_{0}$ and expand the exponential of the former as a power series, that is,

$$
\begin{aligned}
Z_{N, Q}= & \frac{1}{N !} Z_{\mathrm{EM}, Q}(\beta, h) \int \prod_{i=1}^{N} d^{3} \vec{x}_{i} \exp \left(-\beta H_{c}\right) \int_{0}^{0} \prod_{j=1}^{N} D^{3} \vec{\xi}_{j} \exp \left[G^{0}(\vec{\xi})\right] \\
& \times \sum_{n=0}^{\infty} \frac{1}{n !}\left[G_{1}{ }^{D}(\vec{\xi})+G_{2}{ }^{Q}(\vec{\xi})+G_{2}{ }^{F 1}(\vec{\xi})-G_{2}{ }^{2}(\vec{\xi})+O\left(|\vec{\xi}|^{3}, h^{3}\right)\right]^{n} .
\end{aligned}
$$

Step 3. Using the formula

$$
\int_{x(0)=x}^{x(\beta \hbar)=x^{\prime}} D x \exp \left\{-\frac{m}{2 \hbar} \int_{0}^{\beta \hbar} \dot{x}^{2}(t) d t\right\}=\left(\frac{m}{2 \pi \beta \hbar^{2}}\right)^{1 / 2} \exp \left\{-\frac{m}{2 \beta \hbar^{2}}\left(x^{\prime}-x^{\prime 2}\right)\right\}
$$

we can integrate the first term of the series of (B.13) and get

$$
\begin{aligned}
& Z_{N, Q}=\frac{1}{N !} Z_{\mathrm{EM}, Q}(\beta, \hbar) \int \prod_{i=1}^{N} d^{3} \vec{x}_{i} \exp \left(-\beta H_{c}\right)\left\{\left(\frac{m}{2 \pi \beta \hbar^{2}}\right)^{3 N / 2}-Z_{Q}{ }^{\prime}(\vec{x})\right\}, \quad(\mathrm{B} .15) \\
& Z_{Q}{ }^{\prime}(\vec{x})=\prod_{i=1}^{N} \int_{0}^{0} D^{3} \xi_{i} \exp [G(\vec{\xi})] \sum_{n=1}^{\infty} \frac{1}{n !}\left[G_{1}{ }^{D}+G_{2}{ }^{Q}+G_{2}{ }^{F 1}+G_{2}{ }^{F 2}+O\left(|\vec{\xi}|^{3}, \hbar^{3}\right)\right]^{n} .
\end{aligned}
$$

Notice that the first term of (B.15) is the classical partition function. We have to study the order in $\hbar$ of each of the terms of (B.16). For that purpose we use Eqs. (B.4), (B.7), 
(B.11), (B.12), (4.4) and the fact that $\int_{0}^{\beta \hbar} d \tau \sim \beta \hbar$, and get that the contributions of $\left(G_{1}{ }^{D}\right)^{2}, G_{2}{ }^{0}, G_{2}{ }^{F 1}$ and $G_{2}{ }^{F 2}$ go, respectively, with $\beta^{3} \hbar^{2}, \beta^{2} \hbar^{2}, \beta \hbar^{2}$ and $\beta^{2} \hbar^{2}$. Notice that $\left(G_{1}{ }^{D}\right)^{2}$ come from $n=2$ in (B.16). The rest of the terms arising from $n \geqq 2$ and those included in $O\left(|\vec{\xi}|^{3}, \hbar^{3}\right)$ give contributions going with powers of $\hbar$ greater than 2 . Therefore, the first quantum correction is

$$
Z_{Q, 2}^{\prime}(\vec{x})=\prod_{i=1}^{N} \int_{0}^{0} D^{3} \vec{\xi}_{i} \exp \left[G^{0}(\vec{\xi})\right]\left[G_{1}{ }^{D}+G_{2}{ }^{Q}+G_{2}{ }^{F 1}+G_{2}{ }^{2}+\frac{1}{2}\left(G_{1}{ }^{D}\right)^{2}\right]
$$

where subindex 2 recalls the order in $\hbar$.

Step 4. Notice that (B.17), as it stands, is only a formal expression. To evaluate it we write its detailed form, which is the following path integral

$$
\begin{aligned}
Z_{Q, 2}^{1}(\vec{x})= & \int \lim _{M \rightarrow \infty} \prod_{i=1}^{N}\left(\frac{m}{2 \pi \hbar \delta}\right)^{3 / 2} \prod_{l=1}^{M} \frac{d^{3} \vec{\xi}_{i, l}}{\left(m^{-1} 2 \pi \hbar \delta\right)^{3 / 2}} \\
& \times\left[G_{1}{ }^{D}+G_{2}{ }^{Q}+G_{2}{ }^{F 1}+G_{2}{ }^{2}+\frac{1}{2}\left(G_{1}{ }^{D}\right)^{2}\right] \\
& \times \exp \left\{-\frac{m}{2 \pi \hbar \delta} \sum_{i=1}^{N} \sum_{l=1}^{M+1}\left(\vec{\xi}_{i, l}-\vec{\xi}_{i, l-1}\right)^{2}\right\}
\end{aligned}
$$

with

$$
\vec{\xi}_{i, M+1}=\vec{\xi}_{i}(\beta \hbar)=0, \quad \vec{\xi}_{i, 0}=\vec{\xi}_{i}(0)=0, \quad \delta=\frac{\beta \hbar}{M+1},
$$

and where, now,

$$
\begin{aligned}
& G_{1}{ }^{D}=-\frac{q^{2}}{2 \pi \hbar} \sum_{i>j=1}^{N} \sum_{l=1}^{M+1} \delta \frac{D_{i j}^{\alpha}}{\left|\vec{x}_{i}-\vec{x}_{j}\right|} \xi_{i, l, \alpha}, \\
& G_{2}{ }^{Q}=-\frac{q^{2}}{2 \pi \hbar} \sum_{i>j=1}^{N} \sum_{l=1}^{M+1} \delta \frac{\xi_{i, l, \alpha} Q_{i j}^{\alpha \gamma}\left[\xi_{i, l}-\xi_{j, l}\right]_{\gamma}}{\left|\vec{x}_{i}-\vec{x}_{j}\right|}, \\
& G_{2}{ }^{F 1}=-\frac{q^{2}}{\beta \hbar^{2}} \sum_{i=1,2} \int \frac{d^{3} \vec{k}}{(2 \pi)^{3}} \frac{|c(\vec{k})|^{2}}{\omega^{2}} I\left(G_{2}{ }^{F 1}, N, M\right), \\
& G_{2}{ }^{F 2}=\frac{q^{2}}{\beta \hbar^{2}} \sum_{i=1,2} \int \frac{d^{3} \vec{k}}{(2 \pi)^{3}}|c(\vec{k})|^{2} I\left(G_{2}{ }^{F 2}, N, M\right) .
\end{aligned}
$$

$I\left(G_{2}{ }^{F 1}, N, M\right)$ and $I\left(G_{2}{ }^{F 2}, N, M\right)$ are given by

$$
\begin{aligned}
& I\left(G_{2}{ }^{F 2}, N, M\right)=\left|\sum_{l=1}^{N} e^{i \vec{k} \vec{x}_{i}} \sum_{l=1}^{M+1}\left[\vec{\varepsilon}\left(\vec{\xi}_{i, l}-\vec{\xi}_{i, l-1}\right)\right] \vec{k} \frac{\left(\vec{\xi}_{i, l}+\vec{\xi}_{i, l-1}\right)}{2}\right|^{2}, \\
& I\left(G_{2}{ }^{2}, N, M\right)=\left|\sum_{i=1}^{N} e^{i \vec{k} \vec{x}_{i}} \sum_{l=1}^{M+1} \delta \vec{\varepsilon}_{i, l}\right|^{2} .
\end{aligned}
$$

We recall that for the $\vec{\xi}_{i}$ coming from the EM field we are using the midpoint rule (see section 3), so for this $\vec{\xi}_{i}$ we write $\left(\vec{\xi}_{i, 1}+\vec{\xi}_{i, l-1}\right) / 2$ instead of $\vec{\xi}_{i, l}$. Notice also that there are $M+1$ subintervals of length $\delta$ in $[0, \beta \hbar]$ and only $M$ non trivial $\vec{\xi}_{i, l}$ 's. 
To integrate Eq. (B.18) we perform a rotation

$$
\left(\begin{array}{c}
\vec{\xi}_{i, 1} \\
\vdots \\
\vec{\xi}_{i, M}
\end{array}\right)=\left(\begin{array}{ccc}
a_{i, 11} & \cdots & a_{i, 1 M} \\
\vdots & & \vdots \\
a_{i, M 1} & \cdots & a_{i, M M}
\end{array}\right)\left(\begin{array}{c}
\vec{\chi}_{i, 1} \\
\vdots \\
\vec{\chi}_{i, M}
\end{array}\right)
$$

such that the exponent

$$
-\frac{m}{2 \pi \hbar \delta} \sum_{i=1}^{N} \sum_{l=1}^{M+1}\left(\vec{\xi}_{i, l}-\vec{\xi}_{i, l-1}\right)^{2}=-\frac{m(M+1)}{2 \beta \hbar^{2}} \sum_{i=1}^{N}\left(\vec{\xi}_{i, 1} \ldots \vec{\xi}_{i, M}\right) A_{i}\left(\begin{array}{c}
\vec{\xi}_{i, 1} \\
\vdots \\
\vec{\xi}_{i, M}
\end{array}\right)
$$

where $A_{i}$ is the $M \times M$ matrix

$$
A_{i}=\left(\begin{array}{rrrrr}
2 & -1 & 0 & \ldots & 0 \\
-1 & 2 & -1 & \ldots & 0 \\
0 & -1 & 2 & \cdots & 0 \\
\vdots & \vdots & \vdots & & \vdots \\
0 & 0 & 0 & \cdots & 2
\end{array}\right)
$$

diagonalizes to

$$
-\frac{m(M+1)}{2 \beta \hbar^{2}} \sum_{i=1}^{N} \sum_{l=1}^{M} a_{i, l} \vec{\chi}_{i, l}^{2} .
$$

The $a_{i, m l}$ of Eq. (B.26) are the eigenvectors of the matrix $A_{i}$ with eigenvalues $a_{i, m}$. Since the determinant is invariant under unitary transformations we have that

$$
\operatorname{det} A_{i}=\prod_{l=1}^{M} a_{i, l}=M+1
$$

Under the change (B.26) the different $G^{\prime}$ 's, Eqs. (B.20)-(B.25), become functions of $\overrightarrow{\mathcal{X}}_{i, l}$ of the same order as the one they had when expressed in terms of $\vec{\xi}_{i, l}$. Then, to perform the integration we only have to use

$$
\int_{-\infty}^{+\infty} d x x^{2 n} e^{-a x^{2}}=a^{-(n+(1 / 2))} \Gamma\left(n+\frac{1}{2}\right), \quad \int_{-\infty}^{+\infty} d x x^{2 n+1} e^{-a x^{2}}=0 .
$$

In this way we get $G_{1}{ }^{D}$ does not contribute to (B.18) since it is antisymmetric in $\vec{\chi}_{i, l}$.

Before integrating (B.18) we are going to study, up to order $h^{2}$, the influence of the field in the interaction between the particles. For $G_{1}^{F 1}$, see Eqs. (B.22) and (B.24), it is clear that after some algebra

$$
\begin{aligned}
I\left(G_{2}{ }^{F 1}, N, M\right)= & \frac{1}{4} \sum_{i, j=1}^{N} \sum_{l, m=2}^{M} e^{i \vec{k}\left(\vec{x}_{i}-\vec{x}_{j}\right)}\left[\left(\vec{\varepsilon} \vec{\xi}_{i, l}\right)\left(\vec{k}_{\xi i, l-1}\right)\left(\vec{\varepsilon} \vec{\xi}_{j, m}\right)\left(\vec{k}_{\xi} \vec{\xi}_{j, m-1}\right)\right. \\
& -\left(\vec{\varepsilon} \vec{\xi}_{i, l}\right)\left(\vec{k} \vec{\xi}_{i, l-1}\right)\left(\vec{\varepsilon} \vec{\xi}_{j, m-1}\right)\left(\vec{k}_{\xi_{j, m}}\right) \\
& -\left(\vec{\varepsilon}_{\xi, l-1}\right)\left(\vec{k} \vec{\xi}_{i, l}\right)\left(\vec{\varepsilon}_{j, m}\right)\left(\vec{k}_{\xi_{i, m-1}}\right) \\
& \left.+\left(\vec{\varepsilon} \vec{\xi}_{i, l-1}\right)\left(\vec{k} \vec{\xi}_{i, l}\right)\left(\vec{\varepsilon} \vec{\xi}_{j, m-1}\right)\left(\vec{k} \vec{\xi}_{j, m}\right)\right]
\end{aligned}
$$

Suppose $i \neq j$; then we make the change $\vec{\xi}_{i, l} \rightarrow \vec{\xi}_{i, l-1}, \vec{\xi}_{i, l-1} \rightarrow \vec{\xi}_{i, l}$ under which the exponential of Eq. (B.18) is invariant, but for which $I\left(G_{2}{ }^{F 1}, N, M\right)$ vanishes, so only $i=j$ 
contributes to the integral. For $G_{2}{ }^{F 2}$ we have that performing the change (B.26) the exponential of Eq. (B.18) becomes symmetric and $I\left(G_{2}{ }^{F} \cdot N, M\right)$ remains linear in $\vec{\chi}_{i}$ except for $i=j$, where it is quadratic. So by symmetrical integration we have that only $i=j$ contributes. Thus, we have proved that, up to order $\hbar^{2}$, the interaction between different particles via the EM field dissappears, but the self-interaction is still present. This leads us to conclude that, in the classical limit, the interaction between different particles is mediated by the Coulomb potential and not by the EM field, in agreement. with our results in section 2 .

Next, we perform the intergrations in Eq. (B.18). We start with its third term, namely, the one associated to $G_{2}{ }^{F 1}$, which is the leading quantum correction. By making use of Eqs. (B.27), (B.29) with $n=1$ and taking into account that in our case $a^{-1}=\left[2 \beta \hbar^{2}\right]$ $\left.m(M+1) a_{i, l}\right]$, each integration over all $\vec{\chi}_{i, l}$, together with $a^{-1 / 2}$, gives a factor

$$
\left[\frac{2 \pi \beta \hbar^{2}}{m(M+1)}\right]^{3 / 2}\left(\frac{1}{a_{i, l}}\right)^{3 / 2},
$$

so the global factor comes out to be

$$
\left[\frac{m(M+1)}{2 \pi \beta h^{2}}\right]^{3 N / 2} \prod_{i=1}^{N} \prod_{l=1}^{M}\left(\frac{1}{a_{i, l}}\right)^{3 / 2}
$$

which, using Eq. (B.28), is equal to

$$
\left(\frac{m}{2 \pi \beta \hbar^{2}}\right)^{3 N / 2} \text {. }
$$

We still have to deal with the factor $a^{-1}$ of Eq. (B.29), (remember $n=1$ and we have only used $a^{-1 / 2}$ ). For $M=1$ we have that

$$
I\left(G_{2}{ }^{1}, N, M=1\right)=0 .
$$

For $M=2$,

$$
I\left(G_{2}{ }^{F 1}, N, M=2\right)=\frac{1}{2} \sum_{i=1}^{N}\left[\left(\vec{\varepsilon}_{i, 1}\right)^{2}\left(\vec{k}_{\hat{\chi}_{i, 2}}\right)^{2}+\left(\vec{\varepsilon} \vec{\chi}_{i, 2}\right)^{2}\left(\vec{k}_{\chi_{i, 1}}\right)^{2}\right]
$$

where we have used that only $i=j$ contributes, and then we get a factor

$$
\frac{N}{27}\left(\frac{\beta \hbar^{2}}{3 m}\right)^{2} \vec{\varepsilon}^{2} \vec{k}^{2} \text {. }
$$

For $M=3$,

which yields

$$
\begin{aligned}
I\left(G_{2}{ }^{F}, N, M=3\right)= & \frac{1}{4} \sum_{i=1}^{N}\left[\left(\vec{\varepsilon} \vec{\chi}_{i, 1}\right)^{2}\left(\vec{k}_{\vec{\chi}_{i, 2}}\right)^{2}+\left(\vec{\varepsilon} \vec{\chi}_{i, 2}\right)^{2}\left(\vec{k}_{\chi_{i, 1}}\right)^{2}\right. \\
& \left.+\left(\vec{\varepsilon}_{\chi_{i, 1}}\right)^{2}\left(\vec{k}_{\chi_{i, 3}}\right)^{2}+\left(\vec{\varepsilon} \vec{\chi}_{i, 3}\right)^{2}\left(\vec{k}_{\hat{\chi}_{i, 1}}\right)^{2}\right]
\end{aligned}
$$

$$
\frac{N}{8}\left(\frac{\beta \hbar^{2}}{4 m}\right)^{2} \vec{\varepsilon}^{2} \vec{k}^{2}
$$

In general, for any $M$ we have that the factor coming from $a^{-1}$ is

$$
N\left(\frac{\beta \hbar^{2}}{m}\right)^{2}\left(\frac{M-1}{M+1}\right)^{3} \vec{\varepsilon}^{2} \vec{k}^{2} .
$$


Since $\vec{\varepsilon}^{2}(\vec{k}, \lambda)=1$ and $(M-1)^{3} /(M+1)^{3}$ tends to 1 for $M \rightarrow \infty$ we conclude

$$
Z_{Q, 2}^{\prime}\left(\vec{x}, G_{2}{ }^{F 1}\right)=-\left(\frac{m}{2 \pi \beta \hbar^{2}}\right)^{3 N / 2} 2 N \frac{\beta \hbar^{2}}{m^{2}}\left(\frac{q}{c}\right)^{2} \int \frac{d^{3} \vec{k}}{(2 \pi)^{3}}|c(\vec{k})|^{2} .
$$

Analogously we evaluate the corrections going with $\beta^{2} \hbar^{2}$, due to $G_{2}{ }^{Q}$ and $G_{2}{ }^{F 2}$ (second and fourth terms of Eq. (B.18)). The contribution of $G_{2}{ }^{Q}$ goes with the trace of the quadrupole tensor $Q^{i j, \alpha} \alpha$, which vanishes, so

$$
Z_{Q, 2}^{\prime}\left(\vec{x}, G_{2}^{Q}\right)=0 \text {. }
$$

For $G_{2}{ }^{2}$ we have

$$
Z_{G, 2}^{\prime}\left(\vec{x}, G_{1}{ }^{2}\right)=\left(\frac{m}{2 \pi \beta h^{2}}\right)^{3 N / 2} 2 N \frac{\beta^{2} h^{2}}{m} q^{2} \int \frac{d^{3} \vec{k}}{(2 \pi)^{3}}|c(\vec{k})|^{2} .
$$

Finally for $\left(G_{1}{ }^{D}\right)^{2}$, which gives rise to the $\beta^{3} \hbar^{2}$ correction, we get.

$$
Z_{Q, 2}^{\prime}\left(\vec{x},\left(G_{1}^{D}\right)^{2}\right)=\left(\frac{m}{2 \pi \beta \hbar^{2}}\right)^{3 N / 2}\left(\frac{q^{2}}{2 \pi}\right)^{2} \frac{3}{2} \frac{\beta^{3} \hbar^{2}}{m} \sum_{\substack{i>j=1 \\ i>j^{\prime}=1}}^{N} \frac{D^{i j \alpha} D_{\alpha}^{i j^{\prime}}}{\left|\vec{x}_{i}-\vec{x}_{j}\right|\left|\vec{x}_{i}-\vec{x}_{j^{\prime}}\right|} .
$$

For $G_{2}{ }^{F 2}$ and $\left(G_{1}{ }^{D}\right)^{2}$ the factor $(M-1)^{3}$ of $(\mathrm{B} .30)$ is to be replaced by $M^{3}+61 / 6 M^{2}$ $-113 / 3 M+27$ and $M^{3}-5 M^{2}-9 M-4$, respectively.

Notice that $\vec{\varepsilon}^{2} \vec{k}^{2}$ is the only non-trivial invariant under rotations of order four that can be formed out of $\vec{\varepsilon}$ and $\vec{k}$, in agreement with (B.22) and (B.30). Out of $\varepsilon$ the only second order non-trivial invariant under rotation is $\vec{\varepsilon}^{2}$, see Eqs. (B.23) and (B.33). The same happens with $Q^{i j, \alpha}=0$, Eqs. (B.21) and (B.32), and with

$$
\Delta \equiv \sum_{\substack{i>j=1 \\ i>j^{\prime}=1}}^{N} \frac{D^{i j_{\alpha}} D_{a}^{i j^{\prime}}}{\left|\vec{x}_{i}-\vec{x}_{j}\right|\left|\vec{x}_{i}-\vec{x}_{j^{\prime}}\right|}
$$

see (B.20) and (B.34).

By plugging Eqs. (B.31)-(B.35) into (B.18) and, in turn, the latter into Eq. (B.15) we get $(4.5)$, which we were looking for.

Appendix C. Proof of Eqs. (4.9)-(4.11)

From Eqs. (3.15), (3.16), (4.2)-(4.4) it follows that

$$
C+D \underset{\hbar \rightarrow 0}{\rightarrow}-\frac{q}{\omega} \frac{(c \vec{k})}{(2 \pi)^{3 / 2}} \sum_{i=1}^{N} e^{i \vec{k} \vec{x}_{i}} \int_{0}^{\beta \hbar} d \tau\left[\vec{\varepsilon}_{i}(\tau)\right]\left[i \vec{k} \vec{\xi}_{i}(\tau)\right]+O\left(\hbar^{3}\right)
$$

which tells us that the first quantum correction to $\left\langle\vec{A}_{\mathrm{Cl}}(\vec{x})\right\rangle$ and $\left\langle\vec{B}_{\mathrm{Cl}}(\vec{x})\right\rangle$ will come from substituting in Eqs. (3.14) and (3.17) $C+D$ by the right-hand-side of (C.1) and $\exp [G(\vec{y})]$ by $\exp \left[G^{0}(\vec{\xi})-\beta H_{c}\right]$ :

$$
\begin{aligned}
& \left\{\begin{array}{c}
\left\langle\vec{A}_{Q}(\vec{x})\right\rangle \\
\left\langle\vec{B}_{Q}(\vec{x})\right\rangle
\end{array}\right\}=-\frac{Z_{\mathrm{EM}, Q}(\beta, \hbar)}{N ! Z_{N, Q}} \int \prod_{i=1}^{N} d^{3} \vec{x}_{i} \exp \left(-\beta H_{c}\right) \prod_{j=1}^{N} \int_{0}^{0} D^{3} \vec{y}_{j} \sum_{\lambda=1,2} \int \frac{d^{3} \vec{k}}{(2 \pi)^{3 / 2}} \\
& \times\left\{\begin{array}{c}
\vec{\varepsilon}(\vec{k}, \lambda) \\
\vec{k} \wedge \vec{\varepsilon}(\vec{k}, \lambda)
\end{array}\right\} \frac{q}{\omega} c(\ddot{k}) \sum_{i=1}^{N} e^{i \vec{k} \vec{x}_{i}} \int_{0}^{\beta \hbar} d \tau\left[\vec{\varepsilon} \dot{\vec{\xi}}_{i}(\tau)\right]\left[i \vec{k} \vec{\xi}_{i}(\tau)\right] \\
& \times \exp \left[G^{0}(\xi)\right]+O\left(h^{2}\right)
\end{aligned}
$$


both of which vanish since when performing the path integrals we get a result that goes with $\vec{\varepsilon}(\vec{k}, \lambda) \vec{k}=0$. Therefore, we get Eqs. (4.9) and (4.10).

Regarding the electric field (see Eq. (3.18)), we have that, through the same arguments as those used for the quantum partition function,

$$
\begin{aligned}
& \left\langle\vec{E}_{Q}(\vec{x})\right\rangle=\frac{Z_{\mathrm{EM}, Q}(\beta, \hbar)}{N ! Z_{N, Q}}\left(\frac{m}{2 \pi \beta \hbar^{2}}\right)^{3 N / 2} \int \prod_{i=1}^{N} d^{3} \vec{x}_{i} \exp \left(-\beta H_{c}\right) \\
& \left(\vec{V} \sum_{i=1}^{N} \frac{q}{4 \pi\left|\vec{x}-\vec{x}_{i}\right|}\right)\left\{1-\frac{q}{2} N \frac{\beta \hbar^{2}}{m^{2}} \mathscr{C}-3 N \frac{\beta^{2} \hbar^{2} c^{2}}{m} \mathscr{C}+\frac{3 \beta^{3} \hbar^{2}}{m}\left(\frac{q^{2}}{2 \pi}\right)^{2} A+O\left(\hbar^{3}\right)\right\}
\end{aligned}
$$

which leads to Eq. (4.11).

\section{Acknowledgements}

The partial financial support given by Comisión Asesora de Investigación Científica y Técnica, Spain, is acknowledged. One of us (F.R.R.) whishes to express his gratitude to the Department of Theoretical Physies of the University of Zaragoza for hospitality.

\section{References}

[1] I. Bralynicki-Birula, Ann. Phys. (N.Y.) 67, 252 (1971); Acta Phys. Austriaca XVIII, 111 (1977).

[2] F. Ruiz Ruiz and R. F. Alvarez-Estrada, Ann. l'Inst. Henri Poincarè 41, 143 (1984).

[3] G. Dente, Phys. Rev. D 12 (1733) (1975).

[4] H. Stapp, Phys. Rev, D 28, 1386 (1983).

[5] M. B. Halpern and W. Sheqel, Phys. Rev. D 16, 2486 (1977).

[6] F. Rohrlich, "Fundamental Physical Problems of Quantum Electrodynamics", in Foundations of Radiation Theory and Quantum Electrodynamics, edited by A. O. Barut, Plenum Press, New York (1980).

[7] C. Bernard, Phys. Rev. D 9, 3312 (1974).

[8] L. Dolan and R. JACKIw, Phys. Rev. D 9, 3320 (1974); I. Omma, Ann. Phys. (N.Y.) 137, 1 (1981); A. J. Nieni and E. W. Semenoff, Ann. Phys. (N.Y.) 152, 105 (1984); H. Matsumoto. I. OJma and H. Umezawa, Ann. Phys. (N.Y.) 152, 348 (1984); H. Matsumoto, Fortschr. Phys. 25, 1 (1977); M. B. Kislinger and P. D. Morley, Phys. Rev. D 13, 2765 (1976); P. D. Morley, Phys. Rev. D 17, 518 (1978);

[9] R. P. Feymman, Rev. Mod. Phys. 20, 367 (1948).

[10] R. P. Feynman, and A. R. Hibrs, "Quantum Mechanics and Path Integrals", MoGraw-Hill, New York (1965).

[11] B. Simon, "Funetional Integration and Quantum Physics", Academic Press, New York (1979), and references therein.

[12] W. Thirring: "Quantum Mechanics of large systems", Springer, New York (1983).

[13] R. Balescu, "Equilibrium and non-equilibrium Statistical Mechanics", John Wiley \& Sons, New York (1975).

[14] S. Ichimaru, "Basic principles of plasma physics", W. A. Benjamin Inc., Reading, Mass. (1973).

[15] R. P. Feynman, "Statistical Mechanics", W. A. Benjamin Inc., Reading Mass. (1972).

[16] K. Huana, "Statistical Mechanics", John Wiley, New York (1963).

[17] L. S. Schulman, "Techniques and Applications of Path Integration", John Wiley \& Sons, New York (1981). 
[18] L. D. Landau and E. Lifschitz, "Statistical Physies", 3rd edition, part 1, Pergamon Press, London (1980).

[19] W. Wigner, Phys. Rev. 40, 479 (1932); J. G. Kirwood, Phys. 44, 31 (1933); M. Goldberger and E. N. Adams, J. Chem. Phys. 20, 240 (1952).

[20] E. H. Lieb and W. Thirring, Phys. Rev. Lett. 35, 687 (1975); J. M. Combes, R. Schrader and R. Seiler, Ann. Phys. 111, 1 (1978); K. HePP and E. H. Lieb; Phys. Rev. A 8, 2517 (1973).

[21] S. S. Schweber, "An Introduction to Relativistic Quantum Field Theory", Harper and Row, New York (1964).

[22] In preparation. 